\title{
Der Nächste ist ein Anderer. Grenzübergreifende Gemeindepartnerschaften in Berlin und Brandenburg während des Kalten Kriegs
}

\author{
Maria Neumann
}

Between 1945 and 1990, Berlin was the symbolic centre of a divided world. Nevertheless, some links remained intact. For instance, Christian communities aspired to maintain and defend ecclesiastical unity across national borders. But how would that unity be practised? Where did the fault lines run? This essay looks into the relationships of sister churches in East and West Germany. Through a close examination of their connections and inter-relations, it will reveal an interdependent transformation of values and attitudes. Furthermore, I will argue that sister church relationships extended beyond the simplistic notion of "unity". By looking into regular get-togethers of the sister churches, I am able to show that the participating churches underwent continuous change and adjustment as they were exposed to divergent perceptions and expectations. By facing the Other - the other Bloc, the other state, the other Christian - the actors became aware of factual and perceived differences. As a result, they began challenging the status quo in their own churches. Therefore, the patterns of ecclesiastical behaviour in two separate social systems can be regarded as reciprocally affected.

Berlin, Cold War, Communication, GDR, Germany, Histoire croisée, Oral History, Religious Communities

Maria Neumann is a PhD-Student at the Humboldt University Berlin. Her thesis is focused on: Religion in der geteilten Stadt. Christliche Vergesellschaftung und Kalter Krieg in Berlin. Recent Publications: "Wir gehören zusammen!" Christliche Gemeinschaft und kirchliche Zeitungen im geteilten Berlin, in: Deutschland Archiv, 6.12.2017. http://www.bpb.de/geschichte/zeitgeschichte/deutschlandarchiv/259675/wir-gehoerenzusammen-christliche-gemeinschaft-und-kirchliche-zeitungen-im-geteilten-berlin. Religion in der geteilten Stadt. Christliche Vergesellschaftung und Kalter Krieg in Berlin, in: Mitteilungen zur Kirchlichen Zeitgeschichte 11 (2017), S. 115-124. 


\section{Einleitung: Über allem steht die Einheit}

„Wir haben eine deutsche evangelische Kirche!“, verkündete der Berliner Bischof Otto Dibelius wenige Tage nach dem Mauerbau 1961. ${ }^{1}$ Eindringlich warnte er davor, die kirchlichen Kontakte zwischen der Bundesrepublik und der DDR abreißen zu lassen und appellierte an alle ChristInnen, die Einheit der Kirche(n) in Deutschland aktiv zu unterstützen. Doch was machte diese eine - geeinte Kirche aus? Inwiefern ließ sich dieser Anspruch, den sich neben Dibelius viele weitere Kirchenglieder zu eigen machten, im Alltag christlicher Gemeinden diesund jenseits des Eisernen Vorhangs überhaupt praktizieren?

Diese Fragen rücken die Beziehungen zwischen einzelnen Gemeinden aus Ost und West ins Blickfeld, denn sie ermöglichen es, Verflechtungen sichtbar zu machen, die von gegenseitiger Beobachtung, geteilter Kommunikation und wechselseitiger Beeinflussung über Systemgrenzen hinweg zeugen. ${ }^{2}$ Der Begriff Verflechtung, wie ihn Christoph Kleßmann geprägt hat, schließt dabei nicht nur Gemeinsamkeiten oder Unterschiede ein, sondern bleibt gleichsam offen für Konkurrenz- und Abgrenzungsmomente und verweist außerdem auf die permanenten Veränderungen, denen die Beziehungen unterlagen. ${ }^{3}$ Anhand des Aufbaus der Partnerschaften, der Organisation und des Ablaufs von Partnerschaftstreffen oder den dort besprochenen Themen lässt sich zeigen, dass die Gemeinden in der DDR gerade nicht isoliert waren, sondern sich im Partnerschaftsverlauf mit unterschiedlichen Wahrnehmungen und Erwartungen auseinandersetzen und auf Grundlage ihrer gewonnen Erfahrungen immer wieder neu positionieren mussten. Die direkte Konfrontation mit dem Anderen - dem anderen System, dem anderen Staat oder eben dem anderen Christen - sensibilisierte die AkteurInnen für tatsächliche wie gefühlte Unterschiede.

Dem hier versuchten Zugriff auf die Mikroebene der Gemeindepartnerschaften ist jedoch ein Quellenproblem immanent. Nur wenige Quellen sind überhaupt überliefert. Die Kirchenleitungen und Gemeinden in der DDR hinterließen aus Angst vor Überwachung und staatlicher Repression oftmals nur wenige schriftliche Zeugnisse. ${ }^{4}$ Aber auch in den Gemeinden West-Berlins und

1 Dibelius, Otto: „Wie es kam“, in: Dibelius, Otto: Reden an eine gespaltene Stadt, 3. Auflage, Stuttgart 1961, S. 12.

2 Vgl. Kunter, Katharina: „Zwischen Antikommunismus und ökumenischer Idealisierung. Prägende Orte und Milieus für das DDR-Kirchenbild im Westen“, in: epd Dokumentation 14/15 (2013), S. 36.

3 Kleßmann, Christoph: Die doppelte Staatsgründung. Deutsche Geschichte 19451955, Göttingen / Bonn 1982. Siehe auch: Kleßmann, Christoph: „Verflechtung und Abgrenzung - Umrisse einer gemeinsamen deutschen Nachkriegsgeschichte“, in: Klaus Schönhoven / Dietrich Staritz (Hrsg.): Sozialismus und Kommunismus im Wandel. Hermann Weber zum 75. Geburtstag, Köln 1993, S. 486-499.

4 „Weil wir über solche Begegnungen nicht zu viel dokumentieren wollten, steht in 
der Bundesrepublik sind entsprechende Dokumente aus diesem Grund nicht zu finden. ${ }^{5}$ Stattdessen überwiegen Fotografien von Festgottesdiensten anlässlich von Hochzeiten oder Konfirmationen sowie Architekturaufnahmen von sakralen Gebäuden oder Bilder von Ausflügen und festlichen Banketten. ${ }^{6}$ Allein dieser Befund, also die Existenz der zuletzt beschriebenen Quellen einerseits und die Quellenarmut andererseits, macht auf Konfliktlinien aufmerksam, die zwischen alltäglichen und außeralltäglichen Momenten verliefen und dabei zwischen AuBergewöhnlichem und Traditionellem, Öffentlichem und Privatem sowie Eigenem und Anderem unterschieden. Ausgehend davon stützt sich dieser Text neben landeskirchlichen Archivalien und Ego-Dokumenten vor allem auf ZeitzeugInneninterviews, die in den Jahren 2016 und 2017 mit evangelischen und katholischen Gemeindegliedern (Geistlichen und Laien) in Berlin und Brandenburg geführt wurden. Gesine Hefft und der ehemalige Propst der evangelischen Kirche Berlin-Brandenburg, Friedrich Winter, hatten angesichts der Quellensituation schon in den 1990er-Jahren darauf hingedeutet, dass ein Rückgriff auf Methoden der Oral History zur Erforschung von Gemeindepartnerschaften unabdingbar sei. ${ }^{7}$ Denn trotz all der berechtigten Skepsis gegenüber Erinnerungen als Quelle, ${ }^{8}$ liefern die Gespräche mit ZeitzeugInnen doch Hinweise auf Wahrnehmungen und Praktiken, die über die bekannten, oft amtskirchlich geprägten Beschreibungen und Deutungsmuster hinausgehen bzw. diese sogar kontrastieren können.

meinen Kalendern nur eine ganz kurze Notiz (Berlin „Pünktchen') - so hieß die Gaststätte, in der wir das Mittagessen angemeldet hatten.“ Sell, Helmut: „Rückschau auf 25 Jahre Partnerschaft", in: Partnerschaft der Kirchengemeinden Kirn und St. Petri Luckenwalde im geteilten Deutschland von 1978 bis 2004, Broschüre, S. 5.

5 Vgl. Rittberger-Klas, Karoline: Kirchenpartnerschaften im geteilten Deutschland. Am Beispiel der Landeskirchen Württemberg und Thüringen, Göttingen 2006, S. 19-22 und S. 309.

6 Vgl. 1953 - 60 Jahre Partnerschaft - 2013. Im Markgräfler Land Britzingen Dattingen. In Brandenburg Ahrensfelde Mehrow. Broschüre, Pfarreiarchiv Ahrensfelde, ohne Signatur.

7 Vgl. Hefft, Gesine: Was eint uns, wenn uns nichts mehr trennt? Kirchliche Gemeindepartnerschaften vor und nach 1989, Berlin 1996, S. II und S. 23. Sowie: Winter, Friedrich: „Kirchliche Wege zwischen Ost und West (1949-1989). Hinweise auf eine dringende Forschungsaufgabe“, in: Hoburg, Ralf (Hrsg.): Unter dem Dach der Kirche. Festschrift für Joachim Rogge zum 70. Geburtstag, Leipzig 1999, S. 146-147.

8 Auf eine gesonderte Darstellung der methodischen Fallstricke bei der Arbeit mit ZeitzeugenInneninterviews soll hier verzichtet werden. Vgl. Plato, Alexander von: „Interview-Richtlinien“, in: Leh, Almut / Plato, Alexander von / Thonfeld, Christoph (Hrsg.): Hitlers Sklaven. Lebensgeschichtliche Analysen zur Zwangsarbeit im internationalen Vergleich, Wien (u. a.) 2008, S. 443-450. Und: Besier, Gerhard: „Psychologie und Oral History als Faktoren der Sozietät. Anmerkungen zur Akkuratesse von Erinnerung“, in: Kirchliche Zeitgeschichte 1 (1994), S. 102-116. Siehe auch: Rittberger-Klas, S. $23-24$. 


\section{Was ist eine Gemeindepartnerschaft?}

Sowohl von ZeitgenossInnen als auch in der Forschungsliteratur werden unter dem Begriff Kirchenpartnerschaften immer wieder unterschiedliche Kontaktformen zusammengefasst, so dass es schwer ist, den Begriff Gemeindepartnerschaft trennscharf von anderen Partnerschaftsverhältnissen im kirchlichen Bereich zu unterscheiden. ${ }^{9}$ De facto gab es vielfältige Formen kirchlicher Beziehungen zwischen Ost und West, die sowohl auf der Kirchenleitungsebene, als auch zwischen kirchlichen Institutionen wie diakonischen Einrichtungen oder Caritasstellen sowie auf der Ebene der Gemeinden oder zwischen Einzelpersonen angesiedelt waren. ${ }^{10}$ Unter den in dieser Darstellung fokussierten Gemeindepartnerschaften sollen dezidiert bilaterale Paten- und Partnerschaften ${ }^{11}$ zwischen zwei Kirchengemeinden verstanden werden, wobei die entsprechenden AkteurInnen gleichzeitig in andere partnerschaftliche Verhältnisse involviert sein konnten. Das gilt gleichermaßen für die geschilderten Praktiken. Erst die spezifisch basisnahe und zugleich institutionalisierte sowie verstetigte Verbindung bestimmter Charakteristika machte die Gemeindepartnerschaft aus. Diese Definition schließt eine übergemeindliche Koordinierung der Beziehungen nicht grundsätzlich aus. Aber anders als beispielsweise in der thüringischen Landeskirche ist eine solche in Berlin-Brandenburg nicht nachzuweisen; die Gemeinden agierten dort relativ eigenständig. ${ }^{12}$ Die genaue Zahl existierender Gemeindepartnerschaften zu bestimmen ist ohnehin unmöglich, da weder das Bistum Berlin noch die Evangelische Kirche Berlin-Brandenburg die partnerschaftlichen Be-

9 Vgl. u.a. Lepp, Claudia: Tabu der Einheit? Die Ost-West-Gemeinschaft evangelischer Christen und die deutsche Teilung (1945-1969), Göttingen 2005. Siehe auch: Silomon, Anke: Anspruch und Wirklichkeit der „besonderen Gemeinschaft“. Der OstWest-Dialog der deutschen evangelischen Kirche 1969-1991, Göttingen 2006.

10 Beispiele hierfür sind auf evangelischer Seite etwa der Kirchliche Bruderdienst oder die Patenspende. Vgl. Rittberger-Klas, S. 10, S. 55 und S. 66. Auf katholischer Seite sind die Partnerschaften zwischen einzelnen Diözesen oder grenzübergreifende Kontakte des Bonifatiuswerkes beispielhaft zu nennen. Vgl. Homeyer, Josef: „Die katholische Kirche im geteilten Deutschland und ihre Bedeutung für das Zusammengehörigkeitsgefühl“, in: von Hehl, Ulrich / Hockerts, Hans Günter: Der Katholizismus - gesamtdeutsche Klammer in den Jahrzehnten der Teilung? Erinnerungen und Berichte, Paderborn u. a. 1996, S. 24-25.

11 Die Begriffe Gemeindepatenschaft und Gemeindepartnerschaft gehören zum Vokabular der Quellensprache. Während „Patenschaft“ vor allem als ein Begriff galt, den die Kirchenleitungen in der Nachkriegszeit sozusagen von oben zu implementieren versucht hatten, sprachen viele Gemeinden selbst und mit Blick auf dieselbe Beziehung schon früh von „Partnerschaft“.

12 Vgl. Maser, Peter: „Die evangelischen Kirchen in der DDR aus der Perspektive des Westens. Blicke über den Zaun - von der Bundesrepublik aus“, in: epd Dokumentation 14/15 (2013), S. 17. 
ziehungen ihrer Gemeinden systematisch erfasst haben. Diese Tatsache ist zwar in erster Linie auf sicherheitspolitische Praktiken der Kirchen zurückzuführen, aber sie kann gleichsam als ein wichtiges Indiz für die weitgehende Autonomie der Gemeindepartnerschaften in dieser Region gelten. Einen wichtigen Anhaltspunkt im Hinblick auf die Anzahl der bestehenden Beziehungen bietet aber eine 1995 von Hefft durchgeführte Studie über evangelische Gemeindepartnerschaften vor und nach 1989. Hefft hat versucht, mit Hilfe einer Fragebogenaktion so viele Ost-West-Partnergemeinden wie möglich zu eruieren, und die Antworten aus den 881 berücksichtigten Fragebögen qualitativ ausgewertet. ${ }^{13}$ Basierend auf ihren Erhebungen, ergänzt um meine bisherigen Forschungsergebnisse, ist derzeit von 252 nachgewiesenen Gemeindepartnerschaften in Berlin und Brandenburg auszugehen. ${ }^{14}$ Demnach werden als Ost-West-Partnerschaften verstanden: Kontakte zwischen Gemeinden aus Brandenburg oder Ost-Berlin und der Bundesrepublik respektive West-Berlin.

\begin{tabular}{|l|l|l|}
\hline Gemeindepartnerschaften (nach Herkunft) & absolute Zahlen & in $\%$ \\
\hline Brandenburg und West-Berlin & 11 & $4,37 \%$ \\
\hline $\begin{array}{l}\text { Brandenburg und } \\
\text { Bundesrepublik }\end{array}$ & 204 & $80,95 \%$ \\
\hline Ost-Berlin und West-Berlin & 7 & $2,78 \%$ \\
\hline Ost-Berlin und Bundesrepublik & 30 & $11,9 \%$ \\
\hline
\end{tabular}

Abbildung 1: Ost-West-Partnerschaften der Evangelischen Kirche Berlin-Brandenburg $(\mathrm{N}=252)^{15}$

Unter den gelisteten Gemeinden sind auch solche, die mehrere Partnerschaften pflegten, während andere Pfarrsprengel nie Westkontakte hatten und dementsprechend nicht in die Statistik eingegangen sind. Generell gilt, dass die Anzahl der Gemeindepartnerschaften nur bedingt Auskunft über die Qualität (wie Dauer oder Intensität) der Beziehungen geben kann, wenngleich die hohe Zahl nachgewiesener Kontakte darauf schließen lässt, dass es sich um eine verbreitete

\section{Vgl. Hefft, S. 15.}

14 Friedrich Winter bezieht sich auf einen Bericht des Diakonischen Werkes der Evangelischen Landeskirche Baden, wonach es in Berlin-Brandenburg 257 Gemeinden gab, die Kontakte zur Partnerkirche in Baden unterhielten. Vgl. Winter, S. 129.

15 Die Zahlen stützen sich auf die genannte Studie von Gesine Hefft, Beiträge auf der Tagung „Wir sind einander begegnet. Grenzüberschreitende Gemeindepartnerschaften in Ost und West" der Evangelischen Akademie zu Berlin im September 2015, ZeitzeugInneninterviews und Recherchen im Evangelischen Zentralarchiv sowie im Evangelischen Landeskirchlichen Archiv Berlin. 
Beziehungsform handelte. Über katholische Gemeindepartnerschaften liegen keine vergleichbaren Zahlen vor, hier müssen Fallbeispiele hinreichen. Obwohl dieser Text also schwerpunktmäßig evangelische Gemeindepartnerschaften behandelt, sollen auch katholische Quellen berücksichtigt werden. Schon zum jetzigen Zeitpunkt zeichnet sich ab, dass evangelische und katholische Gemeindepartnerschaften zumindest in ihren Ausprägungen durchaus vergleichbar sind und in ihren Praktiken eher durch regionale als konfessionelle Unterschiede geprägt wurden. Dennoch ist darauf hinzuweisen, dass die hier vorausgesetzte Vergleichbarkeit der Konfessionen bereits zeitgenössisch und später auch in der Forschung kritisch kommentiert wurde. So konstatierte etwa der ehemalige Leiter der Zentralstelle Berlin des Deutschen Caritasverbandes, Hellmut Puschmann: „Im Unterschied zur evangelischen Kirche hatten wir also eine starke und enge Verzahnung zwischen Ost und West."“16 Der Kalte Krieg als historischer Hintergrund mag diese Konkurrenz zwischen den Konfessionen hinlänglich verstärkt haben, inwieweit er aber als notwendige Bedingung für diesen Wettbewerb angesehen werden kann, ist fragwürdig. ${ }^{17}$ In der Region Berlin-Brandenburg - an der sogenannten Nahtstelle der Systeme - sind solche Verflechtungen, gerade auch in Abgrenzung zum Anderen, jedenfalls vielfach zu greifen. Denn die Region

symbolisierte und repräsentierte [...] nicht nur den globalen Systemkonflikt, die Teilung der Welt, Europas und Deutschlands, sondern [...] das Wesen, die Strukturen und die Ziele der ihn tragenden antagonistischen Weltsysteme. ${ }^{18}$

Und auch kirchlich handelte es sich um einen besonderen Raum. So blieb das Bistum Berlin staatskirchenrechtlich immer ein geeintes Bistum, das Ost- und West-Berlin umfasste, während die Evangelische Landeskirche Berlin-Brandenburg Ende der 1960er-Jahre in die Bereiche Ost und West unterteilt wurde, die ab 1972 jeweils einem eigenen Bischof unterstanden.

16 Puschmann, Hellmut: „Zur Brückenfunktion des Deutschen Caritasverbandes“, in: von Hehl, Ulrich / Hockerts Hans Günter: Der Katholizismus - gesamtdeutsche Klammer in den Jahrzehnten der Teilung? Erinnerungen und Berichte, Paderborn u. a. 1996, S. 129.

17 Vgl. Rittberger-Klas, S. 193.

18 Lemke, Michael: „Einleitung“, in: Lemke, Michael (Hrsg.): Schaufenster der Systemkonkurrenz. Die Region Berlin-Brandenburg im Kalten Krieg, Köln u. a. 2006, S. 11. 
Maria Neumann

\section{Entstehungskontexte und Motive}

Es ist nicht möglich, die Entstehung von Ost-West-Gemeindepartnerschaften zeitlich exakt einzugrenzen oder genau zu verorten, weil bislang nicht zu klären ist, ob es eine konkrete Anweisung der Berliner Kirchenleitungen gab, Paten- und Partnerschaften aufzubauen. Allein die nicht einheitliche Begriffswahl verdeutlicht das Problem und legt eine orts- wie zeitabhängige Charakterisierung der Beziehungen nahe, die sich überdies verändern konnte: Wann etwa wurden aus Patenschaften Partnerschaften ${ }^{19}$ Einig sind sich HistorikerInnen und ZeitzeugInnen im Hinblick darauf, dass ein enger Zusammenhang zwischen einer vom Hilfswerk der Evangelischen Kirche Deutschlands (EKD) 1949 initiierten Paketaktion und dem Aufbau erster partnerschaftlicher Beziehungen besteht. ${ }^{20}$ Die Initiative basierte auf der Idee, dass die materiell und finanziell zunehmend besser gestellten Gemeinden in Westdeutschland ostdeutsche Gemeinden unterstützen sollten. Im Zuge dieser Hilfsaktion war innerhalb der EKD außerdem darüber beraten worden, dass bestimmte Landeskirchen aus Ost und West künftig als Partnerkirchen intensiver zusammenarbeiten sollten. Infolgedessen kooperierte die Evangelische Landeskirche Berlin-Brandenburg mit den Kirchen Badens, des Rheinlands und Westfalens. ${ }^{21}$ Bei der Auswahl der Partnerkirchen hatte die EKD darauf geachtet, dass sich die Zuordnung nicht allein an den Koordinaten Ost und West orientierte, sondern ebenso älteren Traditionslinien folgte: Bei der Kirche Berlin-Brandenburg und ihren Partnerkirchen handelte es sich um unierte Kirchen. Der Versuch der EKD, die neuen nationalstaatlichen Grenzen zu durchbrechen ${ }^{22}$ sollte die kirchliche Forderung nach der ,Einheit Deutschlands" manifestieren. ${ }^{23}$

19 Winter schreibt dazu, dass 1965 „ohne viel Aufhebens das Wort ,Patenschaft“ in ,Partnerschaft‘ umgewandelt wurde.“ Winter, S. 128. Demgegenüber hält RittbergerKlas beispielsweise fest, dass sich dieser Begriff erst Anfang der 1970er-Jahre durchzusetzen begann, nachdem kirchliche Vertreter aus dem Rheinland 1968 eine entsprechende Umbenennung gefordert hatten. Rittberger-Klas, S. 11 und S. 34.

20 Vgl. 1953-2013. 60 Jahre Partnerschaft. Im Markgräfler Land Britzingen Dattingen. In Brandenburg Ahrensfelde Mehrow, Broschüre, Gemeindearchiv Ahrensfelde Mehrow, ohne Signatur. Siehe auch: Rittberger-Klas, S. 46-47.

21 Vgl. Halbrock, Christian: „Basisarbeit mit der kirchlichen Jugend und Ausbesserungen am Kirchendach. Die Ost-West-Treffen der evangelischen Kirche in der DDR“, in: Deutschland-Archiv 4 (2011), S. 536-545. Siehe auch: Seidel, J. Jürgen: „Neubeginn“ in der Kirche? Die evangelischen Landes- und Provinzialkirchen in der SBZ/DDR im gesellschaftspolitischen Kontext der Nachkriegszeit 1945-1953, Göttingen 1989, S. 189. 22 Dieser Logik entsprechend baute die lutherische Landeskirche Thüringens partnerschaftliche Beziehungen mit Württemberg auf, während die reformierten Gemeinden Mecklenburgs Kontakte nach Emden knüpften, um nur zwei weitere Beispiele zu nennen. Vgl. Rittberger-Klas, S. 208.

23 Bischof Otto Dibelius: „Wir bekennen uns nicht nur zur Einheit Deutschlands, wie 
Doch ganz gleich inwieweit eine wichtige Signalwirkung von der Paketaktion ausgegangen sein mag - überhaupt oder gerade jetzt gemeindepartnerschaftliche Beziehungen aufzubauen -, ein signifikanter Entstehungszeitraum lässt sich davon nicht ableiten. Es scheint vielmehr so, dass in Berlin-Brandenburg eine Verselbstständigung beim Aufbau der Partnerschaften stattgefunden hat und die Kontaktaufnahme nicht unbedingt vom Hilfswerk abhängig geschah. Denn in allen Jahrzehnten der Teilung bis zum Mauerfall und sogar darüber hinaus entstanden neue Partnerschaften.

\begin{tabular}{|l|l|l|}
\hline $\begin{array}{l}\text { Beginn der } \\
\text { Gemeindepartnerschaft }\end{array}$ & $\begin{array}{l}\text { Anzahl der Partnerschaften } \\
\text { (absolut) }\end{array}$ & $\begin{array}{l}\text { Anzahl der } \\
\text { Partnerschaften (in \%) }\end{array}$ \\
\hline Vor 1950 & 13 & $5,16 \%$ \\
\hline 1950er-Jahre & 47 & $18,65 \%$ \\
\hline 1960er-Jahre & 43 & $17,06 \%$ \\
\hline 1970er-Jahre & 43 & $17,06 \%$ \\
\hline 1980er-Jahre & 40 & $15,87 \%$ \\
\hline nach 1989 & 8 & $3,17 \%$ \\
\hline ohne Angaben & 58 & $23,02 \%$ \\
\hline
\end{tabular}

Abbildung 2: Entstehungszeit der Gemeindepartnerschaften

Auch lassen die auf der Ebene der Kirchenleitungen erarbeiteten Konzepte keine Rückschlüsse darüber zu, wie die Gemeinden letztlich zueinander fanden. In vielen Fällen scheint der Kontakt über die Person des Pfarrers oder der Pfarrerin angelaufen zu sein. Die ZeitzeugInnen erwähnten dabei immer wieder die Zufälligkeit der Bekanntschaften. So gab es PfarrerInnen, die sich auf kirchlichen Veranstaltungen kennengelernt hatten, andere, die sich bereits aus gemeinsamen Studienjahren in der Vorkriegszeit kannten und wieder andere, die gemeinsam Dienst in der Wehrmacht getan hatten. Des Weiteren gab es BrandenburgerInnen und Ost-BerlinerInnen, die in die Bundesrepublik geflohen waren und von dort aus versuchten, partnerschaftliche Beziehungen $\mathrm{zu}$ ihren ehemaligen Heimatgemeinden aufzubauen. ${ }^{24}$ Letztlich scheinen auch in diesem Bereich vor allem persönliche Sympathiebeziehungen wesentliche Bedingungen für den Aufbau

wir das mehr als einmal [...] getan haben, sondern wir verwirklichen sie an unserem bescheidenen Teil [...].“, Berlin-Weißensee, 1950, abgedruckt bei: Lepp, S. 108.

24 Vgl. u. a. Interview mit Paul Plume, 2.2.2016, S. 9. Siehe auch: Interview mit A.B., 21.1.2016, S. 1. Außerdem: Sell, Helmut: „Rückschau auf 25 Jahre Partnerschaft“, in: Partnerschaft der Kirchengemeinden Kirn und St. Petri Luckenwalde im geteilten Deutschland von 1978 bis 2004, Broschüre, S. 5. 
einer Partnerschaft gewesen zu sein. ${ }^{25}$ Diese Behauptung wird dadurch gestützt, dass Partnerschaften, die über Anknüpfungspunkte verfügten, durchaus an Antipathien scheitern konnten, wie ein westdeutscher Pfarrer 1995 deutlich zum Ausdruck brachte:

Es war einfach keine gute Idee, in einer Gemeinde, in der so viele DDR-Flüchtlinge leben [...], eine Partnerschaftsbeziehung mit einer DDR-Gemeinde aufzunehmen. [...] Somit entstand nur ein kleines Partnerschäftchen, das von wenigen friedensbewegten jungen Leuten aus unserer Gemeinde getragen wurde. Pfarrer allein können auch keine Partnerschaft garantieren, und die Begegnungen nach der Wende (1989) waren für die Wenigen bei uns enttäuschend, weil die Partner blind (wie seit 40 Jahren gewöhnt) kritiklos der Mehrheit nachliefen. ${ }^{26}$

Nicht nur die im Zitat festgehaltenen enttäuschten Erwartungen der Partnergemeinden, sondern auch die unterschiedlichen Entstehungszeiträume sowie die diversen Möglichkeiten, eine Partnerschaft aufzubauen, deuten darauf hin, dass unterschiedliche Motive im Partnerschaftsverhältnis eine Rolle spielten und sich diese im Zeitverlauf änderten. Prinzipiell kann zwischen christlichen, politischen und typisch intrinsischen Motiven wie Neugier unterschieden werden, wobei sich diese Beweggründe oftmals vermischten und verschieden intensiv wirkten. Winter reflektiert aus der Perspektive der Kirchenleitung folgende Motive: Zentral sei das christliche Gebot zur Nächstenliebe gewesen, daneben hätten diakonische, ökonomische, nationale und pädagogische Gründe die Gemeinden motiviert, entsprechende Kontakte aufzubauen. ${ }^{27}$ Insbesondere das nationale Motiv sei im Laufe der Jahre unter den Vorzeichen des Mauerbaus in den 1960erJahren beziehungsweise der Neuen Ostpolitik unter Willy Brandt in den 1970erJahren hinter die diakonischen und pädagogischen Motive zurückgetreten. Diese seien wiederum zunehmend ökumenisch interpretiert und damit noch mehr aus ihren nationalen Kontexten befreit worden.$^{28}$ Die ökonomische Unterstützung der DDR-Kirchen durch ihre Partner in der Bundesrepublik hatte sich derweil längst auf sämtlichen kirchlichen Ebenen und so auch im Bereich der Gemeindepartnerschaften zur Selbstverständlichkeit entwickelt: Das war allein deshalb notwendig, weil die Kirchen in der DDR bis zum Mauerfall kein tragfähiges

25 Für die Gemeinden Hangelsberg in Brandenburg und Epfenbach in Baden war es, wie für viele andere auch, entscheidend, dass „beide Gemeinden im ländlichen Raum sind.“Fragebogen Epfenbach, ohne Datum. Evangelisches Zentralarchiv (EZA) 172/10. Ein anderer Grund konnte die Übereinstimmung in politischen Fragen sein. Vgl. Interview mit Arnold Löwenbrück, 29.1.2016, S. 2.

26 Pfarrer D. R. an den Protestantischen Landeskirchenrat der Pfalz Dezernat III, Betreff: EKD-Studienprojekt „Verläufe und Chancen von Gemeindepartnerschaften“, 30.5.1995. EZA 172/5.

27 Vgl. Winter, S. 126-127.

28 Vgl. Rittberger-Klas, S. 29-31. 
Konzept entwickelten, das ihnen eine Existenz ohne finanzielle Hilfen aus der Bundesrepublik ermöglicht hätte. ${ }^{29}$

Eine ähnlich differenzierte Analyse ihrer Beweggründe lieferten die einzelnen Gemeindeglieder freilich selten. Sie sprachen neben karitativen und pädagogischen Motiven vor allem von der intrinsischen Motivation zu helfen. Eine Katholikin aus West-Berlin, die jahrzehntelang die Partnerschaft zu einer Ost-Berliner Gemeinde unterstützt hatte, reduzierte ihre Beweggründe auf einen Satz: Sie habe geholfen, weil sie helfen wollte und helfen konnte. ${ }^{30}$ Hier scheint bereits durch, dass nicht nur der Dienst am Nächsten oder das Interesse am Anderen zentral für den Aufbau und die Aufrechterhaltung der Partnerschaften war, sondern der Dienst an sich selbst ebenfalls von Bedeutung war. Beide Partnergemeinden konnten somit von den Verhältnissen profitieren: ideell wie materiell. Zwar wussten die ZeitzeugInnen auch um den gedanklichen Überbau „der besonderen Gemeinschaft" ost- und westdeutscher ChristInnen, doch scheint der Begriff bereits zeitgenössisch zunehmend schlagwortartig verwendet und mehrdeutig interpretiert worden zu sein. ${ }^{31}$ Daneben lassen sich außerdem das Interesse am Fremden, womit keineswegs nur das kirchliche Andere gemeint war, sowie die Hoffnung auf Abwechslung und Abenteuer als mögliche Motive von ost- und westdeutschen PartnerInnen gleichermaßen anführen. ${ }^{32}$ Rückblickend ist es

29 Vgl. Projektskizze, 5.9.1991. EZA 172/5, S. 2. An dieser Stelle ist außerdem darauf hinzuweisen, dass die massiven Unterschiede im Hinblick auf die Finanzkraft der Kirchen auch darauf zurückzuführen sind, wie die Kirchensteuern erhoben wurden. Während die Kirchensteuern in der Bundesrepublik direkt von den Finanzämtern eingezogen wurden und die Kirchen dort als relativ reich galten, war die Abgabe der Kirchensteuer in der DDR freiwillig und erfolgte auf der Basis eines privaten Vereinsbeitrages. Dieses vom Staat gewollte Vorgehen zog ein verhältnismäßig geringes Kirchensteueraufkommen nach sich. Vgl. Großbölting, Thomas: Der verlorene Himmel. Glaube in Deutschland seit 1945, Bonn 2013, S. 52-53. Siehe auch: Maser, Peter: Kirchen und Religionsgemeinschaften in der DDR 1949-1989. Ein Rückblick auf vierzig Jahre in Daten, Fakten und Meinungen, Konstanz 1992, S. 49.

30 Vgl. Interview mit F.J., 21.6.2017, S. 22.

31 „Neben dem Wort an die westlichen Landeskirchen verabschiedete die Regionalsynode Ost [1969] auch ein Wort an die Gemeinden, in dem sie die ,besondere Gemeinschaft‘ der ganzen evangelischen Christenheit in Deutschland bejahte, ,die in einer gemeinsamen Geschichte von Anfechtung und Schuld, Vergebung und Segnung, brüderlicher Hilfeleistung und gegenseitiger kritischer Befragung gewachsen ist'." Lepp, S. 874. Vgl. auch: Rittberger-Klas, S. 37.

32 Aus der Perspektive eines ehemaligen DDR-Bürgers: „Und für uns war's natürlich auch nen Stück westliches Ausland. Das war ne andre Welt. Das war als, wenn Sie mal in nen Ausland gefahr'n sind und ham irgendwas Neues erlebt. Das ist eben bereichernd.“ Interview mit A.B., 21.1.2016, S. 11. Siehe auch: Erste Fahrt des Britzinger Frauenkreises nach Berlin-Ahrensfelde zum Besuch bei der ,Patengemeinde', 1984. Broschüre, Pfarreiarchiv Ahrensfelde, ohne Signatur. 
schwierig, diese diversen Beweggründe zu gewichten. Entscheidend ist, dass ihre Vielzahl eine Beschränkung auf rein religiöse oder in der Kalten-Kriegs-Logik verhaftete systemimmanente Motive definitiv nicht erlaubt.

\section{Gemeinschaftliche Praktiken}

Im Gespräch mit den ZeitzeugInnen über die praktische Gestaltung der Gemeindepartnerschaften sticht eine Tatsache besonders hervor. Aus westlicher Perspektive wurden gemeinhin immer die Hilfsleistungen und damit einhergehende Schwierigkeiten und Verdienste als zentrales Element der Gemeindepartnerschaften herausgestellt. In einigen Fällen kristallisierte sich sogar heraus, dass der Aufbau persönlicher Kontakte zu den Partnergemeinden als irrelevant erachtet wurde. ${ }^{33}$ Diese Anonymisierung oder Abstrahierung des Anderen etwa als Hilfsbedürftigem offenbarte schon zeitgenössisch, was die Gemeindepartnerschaften mindestens auch immer waren: Zweckbeziehungen. ${ }^{34}$ Und nur so lässt sich ferner der Kommentar einer Zeitzeugin verstehen: „Das war eigentlich nicht so, dass mit diesen Leuten in der [Partner-]Pfarrei jetzt ganz enge Freundschaften im Sinne von geistigen Freundschaften entstanden sind. Nein, das war Erledigung von Aufgaben.“35 Allgemein ist bei diesen „Aufgaben“ zwischen materiellen oder finanziellen, geistlichen oder seelsorgerlichen (im Bereich des Kirchlichen) und psychosozialen Hilfen (im Bereich des Weltlichen) zu unterscheiden, die sowohl Einzelpersonen, bestimmten Gruppen in der Gemeinde (etwa den KonfirmandInnen) oder der ganzen Gemeinde zu Gute kommen konnten. ${ }^{36}$ Neben solchen Unterstützungsmaßnahmen schlossen die sogenannten Hilfen die Weitergabe von Informationen mit ein. Es handelte sich also um materielle wie immaterielle Transfers, die - nicht zuletzt abhängig von der jeweiligen Gesetzeslage - auf legalen und illegalen Wegen über den Postweg, von Kurieren oder persönlich erledigt wurden. ${ }^{37}$ Die Hilfen waren nicht gänzlich einseitig ausgerichtet, es gab aber einen deutlichen West-Ost-Überhang. ${ }^{38}$

33 Vgl. u.a. Interview mit F.J., 21.6.2017, S. 21. Siehe auch: Interview mit G.S., 24.6.2017, S. 2.

34 Auf Gemeindepartnerschaften als Zweckbeziehungen wird im Abschnitt über das Ende der Partnerschaften noch genauer eingegangen.

35 Interview mit F.J., 21.6.2017, S. 9.

36 Die materiellen Unterstützungsleistungen reichten von geschmuggelten Printmedien, über Verkehrshilfen, also die Motorisierung von PfarrerInnen auf dem Land, bis hin zu Arbeitsheften für den Konfirmationsunterricht. Vgl. Wanke, Joachim: „Bemühungen um den kirchlichen Zusammenhalt zwischen Ost und West“, in: von Hehl, Ulrich / Hockerts, Hans Günter. Der Katholizismus - gesamtdeutsche Klammer in den Jahrzehnten der Teilung? Erinnerungen und Berichte, Paderborn u. a. 1996, S. 36. Siehe auch: Interview mit F.J., 21.6.2017, S. 9. Oder: Fragebögen, EZA 172/10.

37 Vgl. u. a. Interview mit Prälat Roland Steinke, 23.5.2017, S. 6. Siehe auch: Interview 
Der Nächste ist ein Anderer. Grenzübergreifende Gemeindepartnerschaften

Außerdem sind mit Blick auf die gemeinschaftlichen Praktiken, neben der alltäglichen Korrespondenz zwischen einzelnen Mitgliedern der Partnergemeinden $^{39}$, die Partnerschaftstreffen zu nennen. ${ }^{40}$ Anders als die westdeutschen stellen die Quellen aus der DDR nämlich gerade diese Begegnungstreffen sehr oft in den Vordergrund. Hier fällt bereits auf, dass es den ostdeutschen Gemeinden ein großes Bedürfnis war, auf die Gemeinsamkeiten der Partnergemeinden hinzuweisen, da diese als Gradmesser für das Funktionieren der Beziehungen galten. Dagegen schlossen westdeutsche Partnergemeinden von vorhandenen Ungleichheiten selten auf die Qualität der Beziehungen. ${ }^{41}$

\subsection{Finanzielle und materielle Hilfen: zwischen Notwendigkeit und Selbstverständlichkeit}

In der Quellensprache ist häufig nur dann explizit von finanziellen oder materiellen Hilfen die Rede, wenn kirchliche Amtspersonen sprechen. Einfache Gemeindeglieder reden demgegenüber eher von Geschenken, welche die Westdeutschen ihren ostdeutschen PartnerInnen in geradezu ritualisierter Form per Paket oder im direkten Austausch zukommen ließen. Gleich ob nun von Hilfspaket oder Geschenk die Rede ist, der Gegenstand bleibt in diesem Fall derselbe und nur die Inhalte veränderten sich mit der Zeit. In der unmittelbaren Nachkriegszeit überwog der Versand von Nahrungsmitteln und Gegenständen des alltäglichen Gebrauchs wie Textilien, die in der DDR knapp waren. In den 1960erJahren hingegen verbesserte sich die Versorgungslage in der DDR, was dazu führte, dass sich die Bedürfnisse der Ostdeutschen zunehmend veränderten und individualisierten. Doch bemerkten viele westdeutsche Partnergemeinden diesen Wandel zunächst nicht und sorgten damit für Verstimmungen in den ostdeutschen Gemeinden, die keine „Armenpakete“ mehr empfangen wollten. ${ }^{42}$ Hier zeigt sich, dass sich die Vorstellungen vom Anderen gerade in den mehr oder minder isolierten Jahren nach dem Mauerbau oft nicht mit den Realitäten der Partne-

mit F.J., 21.6.2017, S. 9. Und: Interview mit G.S., 24.6.2017, S. 2. Außerdem zum Paketverkehr und den rechtlichen wie politischen Rahmenbedingungen: Härtel, Christian / Kabus, Petra (Hrsg.): Das Westpaket. Geschenksendung, keine Handelsware, Berlin 2000 .

38 Unter anderem schickten DDR-BürgerInnen Bücher und Kunsthandwerk in die Bundesrepublik und nach West-Berlin. Vgl. Interview mit A.B., 21.1.2017, S. 14.

39 Vgl. Plume, Paul: Briefe in die Freiheit 1988-1990, Ahrensfelde 2009, unveröffentlicht.

40 Vgl. Interview mit A.B., 21.1.2017. Sowie: Interview mit B.K., 11.4.2017, S. 3. Oder Interview mit Paul Plume, 2.2.2016, S. 18 f.

41 „Es gab diese Frage nicht, ob wir ernst genommen werden. Es war de facto so.“ Interview mit Paul Plume, 2.2.2016, S. 12.

42 Rittberger-Klas, S. 100. 
rInnen deckten. Die massiven Informationsdefizite belasteten die partnerschaftlichen Beziehungen und gingen nicht zuletzt mit Solidaritätsverlusten einher. ${ }^{43}$ Der Kontakt zwischen einigen Gemeinden schlief infolgedessen sogar ein oder ruhte zumindest zeitweilig. ${ }^{44}$ Tatsächlich korrigierten viele westdeutsche Gemeinden erst seit den 1970er-Jahren ihre Bilder von der DDR. Die Grundlage dafür war, wie Karoline Rittberger-Klas überzeugend dargelegt hat, vor allem der einsetzende Generationswechsel in den Pfarrhäusern und Gemeindegremien auf beiden Seiten der Mauer. Die Nachkriegsgeneration, die zunehmend kirchliche Ämter und Stellen besetzte, wollte „Erfahrungen der gegenseitigen Entfremdung [nicht länger nur negieren, sondern] verarbeiten.“"45 Die neuen Reisebestimmungen, welche im Zuge des Grundlagenvertrags in Kraft traten, erleichterten diesen Wandel und ermöglichten zumindest Gemeinden aus der Bundesrepublik, sich realistische Einschätzungen vor Ort in der DDR zu machen. In der Folge glichen sich - wenn man so möchte - Angebot und Nachfrage der Hilfen in den 1970er- und 1980er-Jahren wieder an. Arnold Löwenbrück, in den 1980er-Jahren Pfarrer im rheinland-pfälzischen Kirn, erinnert sich: ,Wir schleppten viel über die Grenze: Farbe, theologische Bücher..." ${ }^{\text {"46 }}$ Schon vor seiner Amtszeit hatte sich die Praxis etabliert, dass die Partnergemeinde Kirns, St. Petri in Luckenwalde (Brandenburg), Wünsche äußerte, sobald ein Partnerschaftstreffen geplant wurde. Dabei standen oftmals nicht nur dezidiert kirchliche Objekte auf den Wunschzetteln. Gefragt waren vor allem Mangelwaren wie beispielsweise Musikinstrumente, Badarmaturen, Süßigkeiten oder auch Matchboxautos, die vielfach für kirchliche Einrichtungen bestimmt waren, aber ebenso in die Privathaushalte einzelner Gemeindeglieder übergingen. ${ }^{47}$ Mit der Zeit veränderten sich auch die Ansprüche der DDR-BürgerInnen, was die Qualität der Produkte betraf, wobei sie sich meistens an westlichen Werbesendungen aus Rundfunk und Fernsehen orientierten. Eine West-Berliner Katholikin resümierte:

Ich hab für uns Schokolade von Aldi gekauft, aber wenn's Ostler waren, dann sollte es Milka sein. So, ja? Weil, das kannten sie aus der Werbung. Und wenn ich da dran denke, die [lacht], die Gemeinde Herz Jesu [in Ost-Berlin], die brauchten dringend Mikrophone, glaube ich, für die Kirche. [...] Es musste aber Sennheiser sein, ne ${ }^{48}$

43 Knauft, Wolfgang: Katholische Kirche in der DDR. Gemeinden in der Bewährung. 1945-1980, 2. verbesserte und erweiterte Auflage, Mainz 1980, S. 9.

44 Vgl. Fragebögen, EZA 172/8, EZA 172/10, EZA 172/11 und EZA 172/13.

45 Rittberger-Klas, S. 196.

46 Interview mit Arnold Löwenbrück, 29.1.2016, S. 2.

47 Interview mit A.B., 21.1.2016, S. 1. Siehe auch Interview mit Matthias Kohl, 22.2.2017, S. 20.

48 Interview mit F.J., 21.6.2017, S. 5. 
Die zuweilen als übersteigert wahrgenommenen Wünsche blieben gegenüber der ostdeutschen Partnergemeinde meist unkommentiert. Doch innerhalb der westdeutschen Gemeinden diskutierten Gemeindeglieder angesichts der ostdeutschen Erwartungshaltungen sehr wohl über den Zweck der Partnerschaften. Auch die Kirner Gemeinde stellte die von ihr organisierten Spendenaktionen für Notreparaturen und Instandsetzungsarbeiten an Kirchengebäuden in den 1980erJahren in Frage. Anlass war, dass die Luckenwalder Petri-Gemeinde zuvor um Zuschüsse für den Bau eines neuen Gemeindehauses gebeten hatte. Die drei Partnergemeinden Luckenwaldes, darunter die Gemeinde aus Kirn, stellten insgesamt 90.000 DM für den Neubau zur Verfügung. ${ }^{49}$ Als die Gemeindeleitung in Luckenwalde dann aber beschloss, die Spenden unter anderem für den Einbau teurer Kunstglasfenster zu veranschlagen, reagierten die Gemeindeglieder in Kirn irritiert auf dieses Vorhaben. Wie konnten die Luckenwalder das wenige Geld, das ihnen überhaupt zur Verfügung stand, für kostspielige Glaserarbeiten ausgeben? „Die Kirner verstanden das nicht“, erläutert Pfarrer Löwenbrück, „[aber] die Luckenwalder wollten eben ein schönes Gemeindezentrum haben. “50 Zwar belastete dieser Vorfall das Verhältnis zwischen den Gemeinden nur kurzzeitig, aber er gibt Aufschluss über das Selbstverständnis der Kirner Gemeinde als Förderer und Wohltäter der Luckenwalder Pfarrei.$^{51}$ Denn auch wenn die westdeutschen Gemeinden ihren PartnerInnen offiziell keine Vorschriften machen wollten, war es für sie als SpenderInnen wichtig, nicht ausgenutzt zu werden, was nach Christian Halbrock durchaus vorkam: „Auf die Solidarität aus dem angeblich gefühlskalten Westen antworteten Einzelne aus der vermeintlich solidarischen Ostgesellschaft gelegentlich mit offenem Egoismus. “52 Die Spenden sollten „Sinn“53 machen, und nur was die westdeutschen Partnergemeinden als existenzielle Hilfe verstanden, erschien ihnen lauter.

Frau Löwenbrück, die Ehefrau des Kirner Pfarrers, wies darauf hin, dass das Versenden und Mitbringen von Paketen auch in anderer Hinsicht eine ambivalente Praxis darstellte: „Mir war es peinlich, Care-Pakete zu schicken und es gab dann ein offenes Gespräch darüber. Die Luckenwalder sagten, dass sie darauf

49 Vgl. 120 Jahre St. Petri von 1892-2012, Veröffentlichungen in den Gemeindebriefen 2010-2012 der Evangelischen Kirchengemeinde Luckenwalde, S. 27.

50 Interview mit Arnold Löwenbrück, 29.1.2016, S. 3.

51 „Seit 1978 entstanden mit Hilfe der westlichen Partnerkirchen mehr als 40 kirchliche Gemeindezentren, vor allem in sozialistischen Neubaugebieten." Maser, Peter: Die Kirchen in der DDR, Bonn 2000, S. 11.

52 Halbrock, S. 540.

53 „Die mündlich überlieferte Frage eines Studenten, der in den Semesterferien im Lager des DWW [Diakonisches Werk der evangelischen Kirche in Württemberg] arbeitete, warum man ,Regenschirme in die DDR' schicke, wenn , in der Dritten Welt die Menschen hungern', traf die Stimmung mancher Vertreter der jüngeren Generation.“ Rittberger-Klas, S. 197. 
angewiesen sind.“54 Ihre Aussage zeigt die anhaltende Schwierigkeit, eine gefühlte Patenschaft als gleichberechtigte Partnerschaft zu leben. Diese Problematik kommt des Weiteren darin zum Ausdruck, dass einige ostdeutsche Gemeindeglieder versuchten, sich zu revanchieren, indem sie, wie schon erwähnt, Pakete mit Kunsthandwerk oder Büchern in die Bundesrepublik versandten. Doch hatten diese Pakete in erster Linie einen symbolischen und darüber hinaus mehrdeutigen Wert. ${ }^{55}$ Denn weil DDR-BürgerInnen die persönlichen, in diesem Fall also nicht staatlich oder kirchlich subventionierten Geld- und Sachspenden der westdeutschen PartnerInnen nicht annähernd aufzuwiegen vermochten, manifestierten ihre Geschenke immer auch die Grenzen des Möglichen und damit Ungleichheiten zwischen Ost und West - zwischen sich und den Anderen.

\subsection{Besondere Erlebnisse: direkte Begegnungen der Partnergemeinden}

Wie bereits im vorherigen Abschnitt angeklungen, können auch die persönlichen Treffen der Partnergemeinden nicht ohne einen Blick auf die politischen Rahmenbedingungen betrachtet werden. Dementsprechend sind die Begegnungsmöglichkeiten in unterschiedliche zeitliche Phasen einzuteilen: Bis zum Mauerbau konnten ostdeutsche Gemeindeglieder ihre Partnergemeinden im Westen noch besuchen; umgekehrt reisten westdeutsche Gemeinden in die DDR und nach Ost-Berlin. Danach war der Reiseverkehr in den 1960er-Jahren stark eingeschränkt. Die Kontakte blieben weitestgehend auf den Brief- und Paketverkehr beschränkt. Wenn überhaupt war es nur BürgerInnen aus der Bundesrepublik möglich in die DDR einzureisen, West-BerlinerInnen hingegen blieb der Zugang verwehrt. Erst nach Abschluss des Grundlagenvertrags waren persönliche Treffen in den 1970er-Jahren wieder häufiger möglich. ${ }^{56}$ Allerdings kamen die Partnergemeinden aufgrund der DDR-Reisebestimmungen zunächst ausschließlich in Ost-Berlin zusammen. Es entwickelte sich rasch eine Routine: Die westdeutschen Gemeindeglieder reisten über mehrere, meist drei Tage mit einem Touristenvisum ein, blieben von morgens bis abends in Ost-Berlin, übernachteten aber in West-Berlin. In Ost-Berlin dienten den Partnergemeinden unterschiedliche Orte als Treffpunkte. Neben bestimmten Kirchengemeinden trafen sich die Gruppen in Privatwohnungen, oftmals kirchlicher MitarbeiterInnen, oder in anderen kirchlichen Einrichtungen, wobei für die evangelische Kirche das Stephanusstift in Berlin-Weißensee und für die katholische Kirche das Josefsheim in der Pappelallee zu nennen sind. ${ }^{57}$ Darüber hinaus wurden Großveranstaltun-

\footnotetext{
54 Interview mit Arnold Löwenbrück, 29.1.2016, S. 3.

55 Interview mit Paul Plume, 2.2.2016, S. 14.

56 Vgl. Winter, S. $128 \mathrm{f}$.

57 Vgl. Meckel, Markus: „Geborgenheit und Wagnis“, in Kleßmann, Christoph (Hrsg.): Kinder der Opposition. Berichte aus Pfarrhäusern in der DDR, Gütersloh 1993,
} 
gen wie die Leipziger Messe für Partnerschaftstreffen einzelner Mitglieder genutzt. Nicht zuletzt gab es Treffen in Drittländern, so etwa in Polen oder Ungarn. ${ }^{58}$ Nachdem die Reisebestimmungen weiter gelockert worden waren, besuchten westdeutsche Gemeindeglieder ihre Partnergemeinden auch vor Ort in Brandenburg oder trafen sie in Ausnahmefällen auf größeren kirchlichen Veranstaltungen wie dem Kirchentag in Wittenberg 1983. ${ }^{59}$ Generell verlagerten sich die Begegnungstreffen während der 1970er-Jahre aber vermehrt in die Wohnzimmer einzelner Kirchenglieder und verschwanden damit zunehmend auch aus der innerkirchlichen Öffentlichkeit. ${ }^{60}$ Hintergrund war, dass Kirchengemeinden keine Gruppen in die DDR einladen durften, weshalb Einzelpersonen aus der Gemeinde persönliche Einladungen aussprechen mussten, um diese Bestimmung zu umgehen. Das führte dazu, dass die Gäste fortan in Privathaushalten untergebracht wurden und sich die Zahl der TeilnehmerInnen zudem insgesamt wieder reduzierte. Hier ist festzuhalten, dass es bei dieser Verlagerung der Treffen in Privaträume einerseits darum ging, sich staatlicher Einflussnahme zu entziehen. Andererseits ist darauf hinzuweisen, dass dadurch eine besonders exklusive Atmosphäre in eigentlichen Privatbereichen produziert wurde, die die TeilnehmerInnen von den übrigen Gemeindegliedern unterschied und auch innerhalb der Religionsgemeinschaft zu Anderen machte, die einem ausgewählten Kreis angehörten. ${ }^{61}$ In eine Gemeindepartnerschaft waren also keineswegs alle Personen aus der Gemeinde auch tatsächlich eingebunden; im Gegenteil: Manche wurden dezidiert herausgehalten. ${ }^{62}$ Pfarrer Löwenbrück beschrieb, dass in seinem Fall

S. 101. Siehe auch: Sell, Helmut: „Rückschau auf 25 Jahre Partnerschaft“, in: Partnerschaft der Kirchengemeinden Kirn und St. Petri Luckenwalde im geteilten Deutschland von 1978 bis 2004, Broschüre, S. 6. Außerdem: Rittberger-Klas, S. 109. Und: Interview mit Prälat Roland Steinke, 23.5.2017, S. 9.

$58 \mathrm{Zu}$ diesem Thema wäre ein eigener Forschungsbericht gewiss aufschlussreich. Vgl. Interview mit Prälat Roland Steinke, 23.5.2017, S. 2. Siehe auch: Interview mit B.K., 11.4.2017, S. 2 f.

59 Nicht unterschlagen werden soll, dass ostdeutsche Gemeindeglieder seit den 1980er-Jahren auch immer häufiger ihre Partnergemeinden in der Bundesrepublik besuchten. Gelegenheit dazu boten unter anderem Aufenthalte bei westdeutschen Verwandten. Vgl. Interview mit Paul Plume, 2.2.2016, S. 20.

60 Hiervon zeugt unter anderem die Tatsache, dass es engagierte Kirchenglieder gab, die von den partnerschaftlichen Beziehungen ihrer eigenen Gemeinde keine Kenntnis hatten. Vgl. Interview mit W.T., 24.4.2017, S. 7.

61 „Als alles noch geheimnisvoll zugehen mußte, da gab es oft Mißtrauen gegen den Kreis, der sich mit den ,Westlern' traf.“ Fragebogen Bautzen, ohne Datum, in: EZA 172/ 5.

62 Ob es sich, wie Halbrock meint, bei der Vielzahl dieser Abgrenzungsversuche sogar um eine Bewegung handelte ist hingegen fragwürdig, weil diese exklusiven Kreise innerhalb der DDR isoliert blieben und untereinander keinen Kontakt hatten. Vgl. Halbrock, S. 542. 
meist acht bis zehn Familien zusammenkamen, wobei auf ostdeutscher Seite „intuitiv“ entschieden worden sei, dass nur Personen „ohne Beziehungen zur Staatsmacht" diesem inneren Zirkel angehören sollten. Die westdeutschen TeilnehmerInnen waren in diesen Entscheidungsprozess nicht involviert. ${ }^{63}$ Ferner unterschied sich die Auswahl der TeilnehmerInnen in Ost und West voneinander: In der DDR bestimmten gewöhnlich die Gemeindekirchenräte, wer an Begegnungstreffen teilnehmen durfte. Westdeutsche Gemeindeglieder konnten sich demgegenüber für Fahrten in die Partnergemeinden einfach anmelden. Prinzipiell konnte jedes westdeutsche Kirchenmitglied teilnehmen. ${ }^{64}$ Nichtsdestotrotz galt für beide Gruppen gleichermaßen, dass sie nicht unbedingt als repräsentativ für ihr Herkunftsland zu betrachten waren:

Das Bild der östlichen Christen von der westlichen Kirche wurde geprägt durch die Westler, die man kennenlernte. Das aber waren vorwiegend die aktiven, theologisch und politisch Neugierigen, die irgendwie ,Progressiven', ökumenisch Aufgeschlossenen $[\ldots] .^{65}$

Das galt umgekehrt auch für die ostdeutschen AkteurInnen. Bei vielen von ihnen handelte es sich ebenfalls um Personen, die an den bestehenden Verhältnissen aktiv etwas zu verändern suchten. Doch während einige ihre Ablehnung gegenüber dem DDR-Staat klar zum Ausdruck brachten, ${ }^{66}$ machten andere ZeitzeugInnen deutlich, dass ihre Kritik an bestimmten Missständen in der DDR nicht als oppositionelle Haltung fehlzuinterpretieren sei. Solche Stellungnahmen forderten die Beziehungen schon zeitgenössisch heraus und befeuerten innerkirchliche Loyalitätskonflikte, weil westdeutsche BesucherInnen, sofern es sich nicht um Studierende oder Mitglieder der Evangelischen Jugend bzw. der Katholischen Jugend handelte, oftmals eine Fundamentalopposition ostdeutscher ChristInnen voraussetzten. ${ }^{67}$

63 Interview mit Arnold Löwenbrück, 29.1.2016, S. 2. Auch andere Angaben lassen darauf schließen, dass es sich um sehr kleine TeilnehmerInnenkreise handelte. So habe beispielsweise auch nur „ein Händchen voll“ Katholiken die Gemeindepartnerschaft der West-Berliner Gemeinde St. Ludwig zur Gemeinde Herz Jesu in Ost-Berlin unterstützt. Interview mit F.J., 21.6.2017, S. 3.

64 Vgl. Interview mit A.B., 21.01.2016, S. 9.

65 Vgl. Projektskizze, 5.9.1991. EZA 172/5, S. 2.

66 Vgl. Interview mit Matthias Kohl, 22.2.2017, S. 1 f.

67 Vgl. „Die Bundesrepublik hat sich kaum für'n Osten interessiert. Das ist ja auch politisch so gewesen und damit betraf das auch die Menschen und die war'n dann bei solchen Treffen oder bei solchen Partnerschaften, ham die manchmal gestaunt, was da möglich war. Und wenn die hier war'n, ham sie immer gesagt: ,Ja...ja... euch geht's ja gar nicht so...' [lacht] Als ob sie gedacht haben, sie kommen erst nach Sibirien oder so. [...] Wir konnten das eigentlich nie so richtig verstehen, dass wir [in der DDR] zum Außenseiter gemacht worden sind, obwohl wir uns gar nicht so verstanden haben. Ich hab mich 
Der Nächste ist ein Anderer. Grenzübergreifende Gemeindepartnerschaften

Dessen ungeachtet wurden die Treffen grundsätzlich positiv bewertet, sowohl die Atmosphäre als auch den Ablauf der Begegnungen betreffend: Obwohl in Berlin-Brandenburg von Seiten der Kirchenleitung keine Vorgaben gemacht wurden, folgten die meisten Begegnungstreffen ähnlichen Mustern. Es gab feste Programmpunkte, die in der Reihenfolge variieren konnten, aber dennoch Bestandteile sämtlicher Treffen blieben. Die Begegnungen mit der Partnergemeinde wurden als außeralltägliche, festliche Ereignisse ausgerichtet. Neben informellen Gesprächen fanden Seminare statt, bei denen theologische und kirchenpolitische Fragen im Mittelpunkt standen. Diese wurden zwar bewusst nicht eindeutig von gesamtgesellschaftlichen Inhalten abgegrenzt, sollten aber im Gegenzug nicht als offene politische Diskussionen verstanden werden. Die Mehrzahl der TeilnehmerInnen war vor allem darauf bedacht, vorsichtig zu agieren und nicht die Aufmerksamkeit der Behörden in der DDR zu erregen. ${ }^{68}$ Ein weiteres Indiz dafür, dass diese moderierten Gesprächsrunden in ihrer Bedeutung für den Zusammenhalt der Gruppen nicht überzubewerten sind, liefert die Tatsache, dass die Erinnerungen an die Treffen von anderen Unternehmungen dominiert werden. Abgesehen von kirchlichen Festen erinnerten sich die ZeitzeugInnen hauptsächlich an das kulturelle Rahmenprogramm mit Theater- und Opernaufführungen sowie an Ausflüge ins Umland. ${ }^{69}$ Es scheint fast so, als ob viele ostdeutsche Partnergemeinden ein Programm zusammenstellten, das die vermeintlichen Erwartungen der Westdeutschen kontrastieren sollte. Dabei distanzierten sie sich einerseits keineswegs immer eindeutig von der SED, sondern wiesen unter anderem auf Errungenschaften in der Gesundheits- und Bildungspolitik hin. Andererseits war ihnen offensichtlich daran gelegen, ihren BesucherInnen neue, möglichst parteiferne Perspektiven auf die DDR zu eröffnen. ${ }^{70}$ Die westdeutschen TeilnehmerInnen nahmen diese Differenzierungsversuche durchaus wahr, konnten sie aber nur bedingt nachvollziehen. Entweder weil sie einzig sahen, was sie zu sehen erwartet hatten, oder weil der Anblick des „real existierenden Sozialismus“ ihre zuweilen affirmativen, aber wirklichkeitsfremden Vorstellungen, mit denen sie in die DDR gereist waren, relativierten. ${ }^{71}$ Ähnlich ambivalente Gefühle hatten BesucherInnen aus westdeutschen Gemeinden angesichts der

zum Beispiel sehr... Persönlich, fand ich den Staat besser als den kapitalistischen Staat. [...] Heute lache ich natürlich darüber." Interview mit A.B., 21.1.2016, S. 17 f.

68 Vgl. Interview mit Arnold Löwenbrück, 29.1.2016, S. 3. Siehe auch: Interview mit F.J., 21. 6.2017, S. 9. Und: Interview mit Paul Plume, 2.2.2016, S. 2 f. Davon spricht nicht zuletzt auch das Erstaunen vieler ZeitzeugInnen darüber, dass sie in ihren nach 1990 angeforderten Stasi-Akten keine eklatant negativen Berichte über die Partnerschaftstreffen fanden.

69 Vgl. Rittberger-Klas, S. 110. Sowie: Interview mit G.S., 24. 6.2017, S. 3.

70 Vgl. Interview mit A.B., 21.1.2016, S. 12 f. und S. 18. Interview mit Arnold Löwenbrück, 29.1.2016, S. 2.

71 Vgl. Interview mit Arnold Löwenbrück, 29.1.2016, S. 3. 
Anstrengungen, die Ostdeutsche auf sich nahmen, um ihre Gäste zu bewirten. Die als selbstlos interpretierte Gastfreundschaft ostdeutscher Gemeindeglieder blieb trotz „vollgeladener Tische“72 oft dem stereotypen Bild von der armen DDR verhaftet und erregte Mitleid, wie aus der Schilderung einer Frau aus dem westdeutschen Kirn deutlich wird:

Wir wurden bewirtet wie die Könige. Ich glaube unsere Freunde aus Luckenwalde hatten sich alles vom eigenen Mund abgespart [sic!] was wir genießen durften. Wir wussten um die Not [sic!] die herrschte, was das Besorgen von Lebensmitteln betraf. Ich hatte Hemmungen mich satt zu essen. ${ }^{73}$

Bemerkenswert ist, dass es ostdeutschen Gemeindegliedern umgekehrt genauso ergehen konnte. Paul Plume aus Ahrensfelde (Brandenburg) etwa sah sich in der westdeutschen Partnergemeinde Britzingen mit dem ihm bis dahin unbekannten Phänomen der Altersarmut konfrontiert. Die Rentnerin, bei der er und seine Frau in den 1980er-Jahren untergekommen waren, sei „bettelarm“ gewesen. Die Erfahrung, dass sie in diesem Fall als DDR-BürgerInnen wider Erwarten materiell besser gestellt waren als die westdeutsche Rentnerin, veränderte das Bild des Ehepaares von der Bundesrepublik und stellte gleichzeitig eine systemübergreifende Verständigungsbasis dar. ${ }^{74}$

Eine identitätsstiftende Wirkung für die partnerschaftlichen Verhältnisse hatte daneben die Auseinandersetzung mit einem gemeinsamen Feind: dem Ministerium für Staatssicherheit. ${ }^{75}$ Dieser andere Akteur nahm immer auch Einfluss auf das Partnerschaftsverhältnis bzw. wurde er stets mitgedacht und sei es nur, indem man bewusst versuchte, ihn zu ignorieren. So luden allein Gerüchte und Spekulationen über das tatsächliche Ausmaß der Überwachung oder mögliche Konsequenzen die ohnehin als abenteuerlich und geheimnisvoll beschriebene Atmosphäre zusätzlich auf. ${ }^{76}$ Doch während sich die Partnergemeinden einerseits vor Repressionen fürchteten, fällt auf, dass sie andererseits glaubten, das Risiko

\section{Ebd.}

73 Kreitzer, Isolde: „Begegnung mit der St. Petrigemeinde in Ostberlin“, in: Partnerschaft der Kirchengemeinden Kirn und St. Petri Luckenwalde im geteilten Deutschland von 1978 bis 2004, Broschüre, S. 12.

74 Interview mit Paul Plume, 2.2.2016, S. 12. Susanne Schröder dazu: „Wir hatten ein klares Bild vom ,Westen', wir wussten, dass uns kein Paradies erwartete, wie es bei vielen DDR-Bürgern der Fall war." Schröder, Susanne: Bericht über unsere Gemeindepartnerschaft, Vortrag, https:/www.eaberlin.de/nachlese/chronologisch-nach-jahren/2015/ ost-west-partnerschaft/schroeder-susanne-gemeindepartnerschaft-2015.pdf [08.6.2016, 16:30 Uhr].

75 Vgl. Rittberger-Klas, S. 273-295.

76 Vgl. Interview mit A.B., 21.1.2016, S. 10. In einigen Partnerschaften galt es zudem als abgemacht, dass die TeilnehmerInnen ihre Familienangehörigen nicht über die Treffen informierten. 
kalkulieren zu können: die Ostdeutschen, weil sie den Umgang mit der DDR „gewohnt" waren und die Westdeutschen, weil sie paradoxe Kommunikationsformen voraussetzten und überdies davon überzeugt waren, sich den staatlichen Organen in der DDR im Zweifelsfall entziehen zu können. ${ }^{77}$ An dieser Stelle soll kein Abgleich wahrgenommener Gefahrenlagen mit wirklichen Gefahrensituationen erfolgen, obwohl es durchaus wichtig wäre, danach zu fragen. Dieser Text aber beschränkt sich auf innergemeindliche Perspektiven. Hinzu kommt, dass die AkteurInnen individuelle Repressionenserfahrungen in den allermeisten Fällen nicht mit den Gemeindepartnerschaften in Verbindung brachten. Diese Beobachtung stützt die These, wonach sich die Partnergemeinden in ihrer Selbstwahrnehmung tendenziell als unpolitisch - im Sinne von nicht-aktivistisch verstanden, gleichwohl diese Einstellung weder ihrer Außenwirkung entsprach noch in das Narrativ der kirchlichen Erinnerungskultur einging. Trotz der insgesamt überaus positiven Erinnerungen an die Partnerschaftstreffen blieben die Verhältnisse nicht unbelastet. Es kam nur in den allerwenigsten Fällen zur Entwicklung einer partnerschaftlichen Kommunikation über wahrgenommene Gemeinsamkeiten wie Differenzen. Daraus folgende Zugehörigkeitskonflikte traten spätestens in den Jahren 1989/1990 offen zu Tage.

\section{Grenzen des Gemeinsamen}

Die Grenzen des Gemeinsamen offenbarten sich im Vergleich mit dem Anderen, obschon die Partnergemeinden zunächst nicht nur Unterschiede, sondern ebenso Gemeinsamkeiten feststellten. Insbesondere traditionelle kirchliche Praktiken bildeten Anknüpfungspunkte. Hier wirkten starke Wiedererkennungseffekte und Identifikationskräfte. Der Kirner Pfarrer Michael Zeh wies etwa auf „die alten Choräle und neuen geistlichen Lieder" hin, die ChristInnen aus Ost und West miteinander verbanden. ${ }^{78}$ Die räumliche Verlagerung der Begegnungstreffen von den zentralen Anlaufstellen Ost-Berlins in die ostdeutschen Gemeinden machte

77 „Begegnungen fanden eigentlich, geheim statt“, aber ich bin überzeugt, die Grenzer wussten wo wir hingingen. Das hat man in Kauf genommen. Man hielt sich selbst nicht für so bedeutsam.“, erinnerte sich Löwenbrück. Interview mit Arnold Löwenbrück, 29.1.2016, S. 3. Ähnliche Eindrücke vermittelt der Bericht der Britzinger Pfarrfrau Elisabeth Landes: „Erst kam der aufregende Übergang in die DDR auf dem Bahnhof Friedrichstraße (es wurde etwas gezittert). Alles ging aber ohne Probleme, die Papiere von allen waren in Ordnung und nach dem vielen Kaffee, den wir im Gepäck hatten, fragte niemand. [...] Nun war der Weg frei, der Osten stand uns offen." Erste Fahrt des Britzinger Frauenkreises nach Berlin-Ahrensfelde [1984] zum Besuch bei der „Patengemeinde“, Broschüre, Pfarreiarchiv Ahrensfelde, ohne Signatur, S. 10.

78 Zeh, Michael: „Luckenwalde - Dank für eine Partnerschaft“, in: Partnerschaft der Kirchengemeinden Kirn und St. Petri Luckenwalde im geteilten Deutschland von 1978 bis 2004, Broschüre, S. 22. 
die PartnerInnen auf weitere Gemeinsamkeiten aufmerksam: „Durch die Verteilung auf Privatquartiere ergaben sich noch viele persönliche Gespräche. Wir stellten bald fest, dass die Themen, die uns bewegten, in Pforzheim ebenso aktuell waren. “79 Es entwickelten sich enge persönliche Kontakte wie Freundschaften und sogar Ehen ${ }^{80}$ Aber auch Unterschiede traten zuerst im privaten Umgang hervor. Nicht nur dass die Menschen aus dem anderen Deutschland, wie ZeitzeugInnen immer wieder berichten, anders aussahen, auch die Sprachen der Partnergemeinden unterschieden sich voneinander. ${ }^{81}$ Die Verwendung eigener Begriffe oder Abkürzungen erschwerte die Kommunikation zwischen den Gruppen merklich. ${ }^{82}$ Außerdem waren die Partnergemeinden mit typischen Gruppendynamiken konfrontiert, wobei milieuspezifische Unterschiede genauso eine Rolle spielten wie diverse regionale, nicht zwangsläufig systemimmanente Mentalitäten.$^{83}$ Hinzu kam, dass auch die Frömmigkeitsstile der Gemeinden oft nicht übereinzubringen waren. Überhaupt stellte sich das kirchliche Leben in den Partnergemeinden sehr verschieden dar. Die offenen Gemeinden im Westen, die zur Verwunderung der Ostdeutschen nicht nur „Tanztees und Nähkurse“ anboten, sondern - um ein katholisches Beispiel zu nennen - auch weibliche Ministrantinnen und Lektorinnen zuließen, hatten wenig gemeinsam mit den tendenziell wertkonservativen Gemeinden in der DDR und Ost-Berlin, die an Bibelstunden und Familienkreisen festhielten. ${ }^{84}$ Die Liste der wahrgenommenen Unterschiede und daraus abgeleiteter Probleme ließe sich weiter fortsetzen. $\mathrm{Zu}$ nennen wären beispielsweise das generelle „Fehlen gemeinsamer Themen“, die „Überalterung der Partnergemeinde“, also Altersunterschiede, Probleme nach

79 Schröder, Susanne: Bericht über unsere Gemeindepartnerschaft, Vortrag, https:// www.eaberlin.de/nachlese/chronologisch-nach-jahren/2015/ost-west-partnerschaft/schroe der-susanne-gemeindepartnerschaft-2015.pdf [08.06.2016, 16:30 Uhr].

80 Allein aus der Partnerschaft zwischen den evangelischen Gemeinden Babelsberg und Langensteinbach gingen beispielsweise sieben Ost-West-Ehen hervor. Vgl. Przybilski, Helmut: Erfahrungen mit Ost-West-Partnerschaften, Vortrag, https://www.ea berlin.de/nachlese/chronologisch-nach-jahren/2015/ost-west-partnerschaft/przybilskihelmut-ost-west-partnerschaften-2015.pdf [8.6.2016, 16:53 Uhr].

81 Im Fall Berlins fiel etwa der Dialekt besonders auf. Während es in West-Berlin verpönt war, den Berliner Dialekt zu sprechen, war dieser in Ost-Berlin sehr gebräuchlich. Vgl. Interview mit F.J., 21.6.2017, S. 20. Siehe außerdem: Interview mit Siegfried Schmidtmann, 2005, Oncken-Archiv, ohne Signatur, S. 13. Siehe auch Interview mit Paul Plume, 2.2.2016, S. 10. Und: Rittberger-Klas, S. 226.

82 Vgl. Interview mit A.B., 21.1.2016, S. 13. Interview mit Arnold Löwenbrück, 29.1.2016, S. 3.

83 Vgl. Erste Fahrt des Britzinger Frauenkreises nach Berlin-Ahrensfelde zum Besuch bei der ,Patengemeinde', 1984. Broschüre, Pfarreiarchiv Ahrensfelde, ohne Signatur. Oder: Interview mit Paul Plume, 2.2.2016, S. 10.

84 Vgl. Lepp, S. 512. Oder: Interview mit A.B., 21.1.2016. Siehe auch: Interview mit F.B., 21.6.2017, S. 14. 
Pfarrstellenwechseln, Neidvorwürfe oder Konkurrenzen westdeutscher Gemeinden um ostdeutsche Partner, sowie ganz pragmatisch: die weiten Entfernungen zwischen den Partnergemeinden. ${ }^{85}$

Von eigentlichem Interesse soll hier aber etwas anderes sein, nämlich die Frage, wie die Partnergemeinden mit diesen Differenzen umgingen, wie sie also auf den Nächsten, der ein Anderer war, reagierten. Zusammengefasst lassen sich drei Strategien eruieren. Die erste Möglichkeit beruhte auf Akzeptanz und Verständnis und bedeutete, Unterschiede einstweilen anzuerkennen, nicht beseitigen zu wollen, und irgendwie produktiv werden zu lassen. ${ }^{86}$ Dabei versuchten gerade Gemeinden, die diesen Weg wählten, spätestens ab den 1970er-Jahren nicht mehr nur auf Vergangenes und Traditionelles zu rekurrieren, sondern bewusst nach neuen Anschlussmöglichkeiten zu suchen, was aber nicht zwangsläufig eine Auseinandersetzung mit den bekannten Problemen nach sich zog. Weiterhin wurde die wahrgenommene Andersartigkeit immer wieder in irreführenden Bildern dargestellt. So beschrieben westdeutsche Gemeindeglieder ChristInnen aus der DDR zum Beispiel noch in den 1980er-Jahren als „,exotisch“ “. ${ }^{87}$ Die damit einhergehende Festlegung auf das Außergewöhnliche verhinderte gleichzeitig eine möglichst gleichberechtigte Kommunikation zwischen den Gemeinden. Zweitens bestand die Möglichkeit einer bewussten Einflussnahme auf den Anderen, die darauf abzielte, die wahrgenommenen Differenzen zu glätten, einander näher zu rücken und gleicher zu werden. Dementsprechend gab es Versuche, erzieherisch auf die Partnergemeinde einzuwirken, Lehrtätigkeiten zu übernehmen und vermeintlich überzeichnete oder falsche Bilder und Vorstellungen zu korrigieren. Im Gespräch miteinander hofften viele Gemeindeglieder, die Partnergemeinden für ihre spezifische Situation zu sensibilisieren. Die Reaktionen darauf waren in Ost und West sehr unterschiedlich. Während einige Gemeindeglieder darauf drangen, noch mehr zu erfahren und ihr Wissen zu erweitern, wurde aus anderen Kreisen der Vorwurf der Bevormundung laut. Meist schlugen die Anstrengungen dann fehl, wenn westdeutsche Gemeinden ihre ostdeutschen PartnerInnen aufforderten, das eigene Verhältnis zum Staat kritisch zu hinterfragen. Denn anstatt sich zu erklären, rechtfertigten sich die Ostdeutschen oftmals, was dazu führte, dass sich die vormals aufgeweichten Fronten wieder ver-

85 Vgl. Fragebögen, EZA 172/10.

86 „Das war eigentlich auch logisch, weil im Osten hat, hatte man [...] nen gemeinsamen Feind. Und dann bleibt man natürlich in seinen Traditionen und [...] im Gegenteil man verstärkt sie dann noch. Während man im Westen natürlich neue Wege suchte, damit auch moderne Menschen sich kirchlich engagieren konnten. [...] Ich oder wir haben gesagt diese Form der Frömmigkeit, wie man sie im Osten aus Traditionsgründen, aus guten Gründen pflegte, die nehmen wir nicht auf. “Interview mit F.J., 21.6.2017, S. 7 und S. 14.

87 Interview mit Paul Plume, 2.2.2016, S. 2. 
härteten ${ }^{88}$ Drittens konnte es sein, dass den Partnergemeinden eine Überwindung der Differenzen beziehungsweise ein gemeinsamer Umgang damit unmöglich erschien und sie eine Partnerschaft infolgedessen ablehnten: Sie fühlten sich unverstanden und blieben verständnislos. In diesem Zusammenhang stand neben anderen Ursachen häufig auch der Vorwurf der Arroganz im Raum. ${ }^{89}$ Ostdeutsche warfen westdeutschen Gemeinden vor, sich ihnen gegenüber überheblich oder selbstgerecht zu verhalten. Umgekehrt bemerkten westliche PartnerInnen, dass sich die Mitglieder in den Ostgemeinden als "die ,wahren Christen“ betrachteten, während im „Westen die Liberalen waren, die die Sache so langsam aufweichten." ${ }^{\circ 0}$

Gemein ist allen drei Optionen, dass sie auf unterschiedliche Verflechtungsmomente innerhalb der partnerschaftlichen Verhältnisse verweisen, gleich ob die Partnergemeinden zum Ausdruck brachten, an den Beziehungen festhalten zu wollen, oder ob sie Entfremdungs- und damit Entflechtungstendenzen feststellten, die ein weiteres Miteinander zunehmend unwahrscheinlich erscheinen lieBen. Damit belegen diese gleichzeitigen ungleichen Entwicklungen der Beziehungen letztlich, dass sich auch die grenzüberschreitenden Gemeindepartnerschaften nur schwer unter einem bestimmten (Einheits-)Begriff subsumieren lassen. Anders stellt sich das auf der praktischen Ebene dar, denn das Schweigen etablierte sich in all diesen Verflechtungen als zentrale Praxis und gewährleistete auf diese Weise eine stabile Konstruktion des Anderen: Wenn es darum ging, die sensiblen Beziehungen aufrechtzuerhalten, unterdrückten die Partnergemeinden etwaige Kritik. Denn dass sie vieles besprachen, bedeutete eben nicht, dass auch alles angesprochen werden konnte. ${ }^{91}$ Durch das Schweigen hingegen wurde eine Gemeinsamkeit impliziert, die erst beim Sprechen darüber fragil zu werden drohte. Aber geschwiegen wurde auch dort, wo Gemeindeglieder glaubten, dass es den Partnerschaften generell an einer gemeinsamen Basis fehle. Das Schweigen bestätigte sie nicht nur in ihrer Haltung, sondern schloss darüber hinaus eine Auseinandersetzung mit wahrgenommen Unterschieden aus und im Zuge dessen auch die Infragestellung oder die Veränderung vorgefertigter Meinungsbilder. Entgegen der eigentlichen Intention, aber letztlich wenig überraschend, wirkte sich dieses Schweigen zuweilen deeskalierend aus, weil es eben oft nicht zum offenen Konflikt kam und die Partnerschaften infolgedessen „trotzdem“ weiter existierten. Diese Konstellationen gerieten erst 1989/1990 ins Wanken. Bis dahin beendeten nur vier von den 252 nachgewiesenen Partnerschaften, die Ost-Berliner oder Brandenburger evangelische Gemeinden mit Gemeinden aus WestBerlin oder der Bundesrepublik führten, ihre Beziehungen offiziell. Und erst danach stieg die Zahl der Trennungen rasant an. Die zuvor vermiedenen wie

88 Vgl. Interview mit A.B., 21.1.2016, S. 20.

89 Vgl. Rittberger-Klas, S. 227.

90 Interview mit F.J., 21.6.2017, S. 7.

91 Vgl. Winter, S. 138. 
vermeidbaren Konfliktthemen im Hinblick auf kirchenpolitische Fragen oder Frömmigkeitsstile wurden jetzt virulent: ${ }^{92}$ Als gleiche Mitglieder der wiedervereinten EKD oder Deutschen Bischofskonferenz waren die Partnergemeinden strukturell rasch wieder eng miteinander verwoben worden, weshalb auch sie jenen Debatten nicht länger ausweichen konnten, die die Moderne herausgefordert und der Kalte Krieg paradoxerweise genährt und gleichzeitig erübrigt hatte.

\section{Getrennt, aber wiedervereint: Das Ende vieler Partnerschaften nach 1989/1990}

Warum aber trennten sich so viele Partnergemeinden angesichts der neuen Herausforderungen gerade im Moment der staatlichen und der mit ihr einhergehenden kirchlichen Wiedervereinigung?

Die meisten Partnergemeinden reagierten auf den Mauerfall zunächst euphorisch. Viele Gemeindepartnerschaften erlebten unmittelbar nach 1990 einen Aufschwung..$^{33}$ In den Tagen und Wochen nach dem 9. November $1989 \mathrm{kam}$ es immer wieder zu Spontanbesuchen ostdeutscher Partnergemeinden in der Bundesrepublik und West-Berlin. ${ }^{94}$ Ostdeutsche Gemeinden konnten nun endlich auch als Gruppen in den Westen reisen und die Zahl der Teilnehmenden bei diesen Begegnungstreffen schnellten nach oben. Doch anstatt der anfänglich positiven Gefühle setzte schon bald darauf eine große Ernüchterung ein: Der personelle Zustrom, den sich die ostdeutschen Kirchen nach 1990 erhofft hatten, blieb aus. Vor allem die ostdeutschen Gemeinden verloren im Wettbewerb mit anderen, neuen Freizeitangeboten, die kaum mehr an kirchliche Einrichtungen gebunden waren. ${ }^{95}$ Die Reisefreiheit erwies sich dabei als ein entscheidender Faktor. Denn für die Ostdeutschen stellten die westdeutschen Partnergemeinden nunmehr keinen exklusiven Kontakt ins Ausland mehr dar. Gerade den westlichen Partnern fiel es besonders schwer, dieses Selbstverständnis aufzugeben.

92 Gesine Heffts Studie aus dem Jahr 1995 belegt das plausibel. Vgl. Hefft, S. 44-61.

93 Das Verhältnis ostdeutscher Christen zur Opposition in der DDR in den 1980erJahren ist ein eigenes Forschungsthema, das hier nicht bearbeitet werden kann. Für die Gemeindepartnerschaften scheint zu gelten, dass das Thema sowohl in der Korrespondenz, als auch bei persönlichen Treffen keine besondere Rolle gespielt hat. Vgl. unter anderem Interview mit Paul Plume, 2.2.2016, S. 21 f.

94 Vgl. Interview mit A.B., 21.1.2016, S. 19. Oder: Interview mit F.J., 21. 6. 2017, S. 11. 95 „Aber bei aller Freude, der Ansturm der verlorenen Gemeindeglieder bleibt aus. Es gibt außerhalb der Kirche so viele Möglichkeiten sich zu entfalten, dass die Kirche nicht der gewünschte Sammelpunkt war. Auch zogen viele von Luckenwalde aus beruflichen Gründen oder Studium weg oder hatten durch den weiten Weg zur Arbeit weniger Möglichkeiten sich zu engagieren." Bohmann, Wolfgang: 120 Jahre St. Petri, Vortrag, Luckenwalde 2012, S. 17. 
Viele reagierten enttäuscht auf die sich verändernden Interessen und das als Undankbarkeit wahrgenommene Verhalten der ostdeutschen Partnergemeinden. Pfarrer Löwenbrück beschrieb den Blick der Kirner Gemeinde auf den Partner in Brandenburg: „Die Luckenwalder fuhren [jetzt] nach Schweden, statt nach Kirn. [...] Das hat an der Ehre gekratzt. [Aber] man hat das nicht sagen können, man konnte das ja verstehen. " ${ }^{96}$ Gleichzeitig machten die ostdeutschen Gemeinden die westdeutschen Partner für das Abflachen der Beziehungen verantwortlich. Aus ihrer Perspektive spielte es eine erhebliche Rolle, dass sich die Alltagsprobleme der Partnergemeinden anzugleichen schienen und das Leben der Anderen dem Eigenen damit ähnlicher wurde. Die Tatsache, dass die zuvor noch als ,exotisch“ charakterisierten Ostdeutschen plötzlich als Gleiche galten, habe die Beziehungen für die Westdeutschen uninteressant gemacht. Während die Gemeinden Schwierigkeiten in der Partnerschaft vor dem Mauerfall oftmals auf die bestehenden Unterschiede zurückführten, sahen sie die Ursache für das Auseinanderbrechen der Beziehungen nun in ihren Gemeinsamkeiten. Ausdruck fand diese Einschätzung auch darin, dass viele Gemeinden zeitgleich neue Partnerschaften, meist in weit entfernten Entwicklungsländern, aufbauten. ${ }^{97}$ Darüber hinaus wurde von Seiten der ostdeutschen Gemeinden der Vorwurf laut, in den Jahren der Teilung wie bloße Hilfeempfänger behandelt worden zu sein. Sie unterstellten, dass es nicht im Interesse ihrer PartnerInnen gewesen sei, einander auf Augenhöhe zu begegnen, sondern Abhängigkeitsverhältnisse zu schaffen. Der Blick auf die Partnerschaften veränderte sich somit auch aus den Gemeinden heraus und wurde von diesen - oftmals nachträglich - politisch aufgeladen. Sich in dieser zunehmend vorwurfsvollen Atmosphäre zu bewegen, wurde immer unangenehmer und schwieriger. Vielen Partnergemeinden gelang es nicht, die Beziehungen wieder zu stabilisieren. Einige trennten sich abrupt und wortlos, andere beendeten die Partnerschaft mit einem offiziellen Festakt. ${ }^{98}$ Bemerkenswert ist aber, dass keineswegs alle partnerschaftlichen Kontakte abbrachen. Die Gemeinden Michendorf und die Melanchthongemeinde in Pforzheim oder die Gemeinden in Ahrensfelde/Mehrow und Britzingen führen ihre Partnerschaften beispielsweise bis heute fort. Trotz des Generationenwechsels haben sich viele Praktiken in diesen Beziehungen nicht geändert. Die Gemeindepartnerschaften zeichnen sich weiterhin durch regelmäßige gegenseitige Besuche, den Austausch der Gemeindebriefe und die monatliche Fürbitte für die Partnergemeinde im Gottesdienst aus. Inhaltlich wurden die Partnerschaften insofern neu ausgelotet, als dass „,nunmehr das aktuell Gemeinsame reflektiert wird und da schließt sich der Kreis gemeinsamer Wertungsmuster: Was ist eigentlich christlich? [...] Das

96 Interview mit Arnold Löwenbrück, 29.1.2016, S. 2 f.

97 Vgl. Interview mit A.B., 21.1.2016, S. 23.

98 Sell, Helmut: Rückschau auf 25 Jahre Partnerschaft, in: Partnerschaft der Kirchengemeinden Kirn und St. Petri Luckenwalde im geteilten Deutschland von 1978 bis 2004, Broschüre, S. 7. 
hat sehr wohl eine politische Handlungskomponente in allen Bundesländern. “99 „Überlebt“ haben folglich die Partnerschaften, die bis heute Antworten auf systemübergeordnete Fragen suchen, wie etwa nach der Rolle von Religion in modernen, säkularisierten Gesellschaften.

\section{Der Nächste ist immer ein Anderer}

Was wissen wir, wenn wir uns mit Gemeindepartnerschaften im Kalten Krieg beschäftigen? Warum ist es sinnvoll, sich mit diesen deutsch-deutschen Beziehungen auseinanderzusetzen?

Zunächst einmal sollte deutlich geworden sein, dass auch der Nächste immer ein Anderer ist. Diese Feststellung ist deswegen nicht einfach banal, weil sie das Bild, das die Kirchenleitungen von der einen, nicht gleichen, sondern selben Kirche gezeichnet hatten, wenn nicht korrigiert, so doch schattiert. Die Forschung, die lange Zeit versucht war, sich dem bekannten Narrativ anzuschließen, ist somit angehalten, innerkirchliche Differenzierungen zumindest stärker in Betracht zu ziehen. Denn es öffnen sich neue Perspektiven, wenn mit Blick auf die christlichen Gemeinschaften zwischen privatem und (teil-)öffentlichem oder kirchenleitendem und basisorientiertem Handeln unterschieden wird. In diesem Zusammenhang stellte der Kalte Krieg einen wichtigen Hintergrund dar. Er isolierte die Gemeinden aber nicht vollständig, weshalb grenzübergreifende Verflechtungen erst recht genauer zu betrachten sind: Es fand Kommunikation über die Mauer hinweg statt, auch wenn KatholikInnen und ProtestantInnen, Ost- und Westdeutsche, Geistliche und Laien dabei mit verschiedenen Einheitsbegriffen operierten und diese über den gesamten Zeitraum hinweg unterschiedliche Ergebnisse hervorbrachten. Schon sehr bald nach Kriegsende bildeten die Gemeinden weniger eine Einheit, als dass sie einander verbunden waren. Deshalb war für sie längst Alltag, was auf Kirchenleitungsebenen bis 1989 konsequent vermieden wurde: Die Gemeinden unterschieden nicht nur zwischen sozialen und politischen Wirklichkeiten oder verschiedenen Herkunftsmilieus, sondern bemühten sich darüber hinaus aktiv einen Umgang mit den ständig wechselnden Verhältnissen von Nähe und Distanz zu finden, wobei gerade das Changieren zwischen diversen und unterschiedlich wahrgenommenen Abhängigkeiten einerseits und der vorangenommenen Gleichheit der Gemeinden andererseits viele Partnerschaften letztlich stabilisierte. Das war auch möglich, weil die Partnergemeinden an der Basis agierten und dementsprechend losgelöst von jenen amtskirchlichen Diskursen, die nur mehr begrenzt alltagstaugliche Identifikationsmöglichkeiten anbieten konnten. Der beobachtete Rückzug der Ge-

99 Schröder, Susanne: Bericht über unsere Gemeindepartnerschaft, Vortrag, https:// www.eaberlin.de/nachlese/chronologisch-nach-jahren/2015/ost-west-partnerschaft/schroe der-susanne-gemeindepartnerschaft-2015.pdf [08.6.2016, 16:30 Uhr]. 
meinden ins Private stützt diese These. Dauerhafte Verflechtungen auf der Gemeindeebene basierten auf persönlichem Austausch und Empathie sowie konkreten Hilfsmaßnahmen, während Beziehungen, die diesen Ansprüchen nicht genügten, etwa weil sich die Partnergemeinden zunehmend entfremdeten, scheiterten bzw. auf Zweckbeziehungen reduziert wurden. Dabei ist auch zu berücksichtigen, dass die Partnerschaften nur eine sehr geringe Öffentlichkeitswirkung erreichten, was den Charakter der Beziehungen ebenfalls kennzeichnete. Zum Einen führte das dazu, dass die Gemeinden stark auf sich selbst zurückgeworfen waren und sich neue Räume erschließen mussten. Dort aber war es offenbar möglich, sich Systemfragen in einem gewissen Maß zu entziehen, was wiederum eine innergemeindliche Entlastung bedeuten konnte. Zum Anderen verweist dieser Umstand darauf, dass die politische Dimension der Beziehungen keineswegs eindeutig bestimmt werden kann und vorerst im Spekulativen verbleiben muss, obwohl der Drang der Forschung nach Eindeutigkeit hier besonders groß zu sein scheint. An dieser Stelle lässt sich konstatieren, dass der Widerspruch von Fremd- und Selbstbeschreibungen nicht nur die Kontakte zwischen Ost und West irritierte, sondern als Muster auch konfessionsübergreifende und selbst innerkirchliche Beziehungen innerhalb eines Landes prägte. Der dynamische Wandel von Einstellungen und Werten wirkte dies- und jenseits der Mauer und konnte wechselseitig die Logiken kirchlichen Handelns in zwei unterschiedlichen Gesellschaftssystemen beeinflussen.

Was wir nicht wissen, ist, inwieweit die deutsch-deutschen Gemeindepartnerschaften im Vergleich zu Partnerschaften zwischen Gemeinden aus der DDR beziehungsweise der Bundesrepublik und Drittländern eine besondere kommunikative Qualität erreichten. Denn die nachweislich engen Beziehungen zwischen evangelischen Gemeinden aus der DDR und den Niederlanden, in denen intensiv zu Themen wie Entkirchlichung oder Privatisierung der Religion gearbeitet wurde, lassen auch die deutsch-deutschen Partnerschaftsverhältnisse in einem anderen Licht erscheinen. ${ }^{100}$ Dem Nächsten stand möglicherweise ein Anderer noch näher.

\section{Literaturverzeichnis}

120 Jahre St. Petri von 1892-2012, Veröffentlichungen in den Gemeindebriefen 20102012 der Evangelischen Kirchengemeinde Luckenwalde.

Besier, Gerhard: „Psychologie und Oral History als Faktoren der Sozietät. Anmerkungen zur Akkuratesse von Erinnerung“, in: Kirchliche Zeitgeschichte 1 (1994), S. 102116.

100 Hogebrink, Laurens: Die armen Verwandten - oder der bessere Teil Deutschlands? Was haben wir voneinander gelernt, was hat uns an den DDR-Kirchen beeindruckt oder irritiert?, in: epd Dokumentation 14/15 (2013), S. 27-31. 
Der Nächste ist ein Anderer. Grenzübergreifende Gemeindepartnerschaften

Bohmann, Wolfgang: 120 Jahre St. Petri, Vortrag, Luckenwalde 2012.

Dibelius, Otto: „Wie es kam“, in: Dibelius, Otto: Reden an eine gespaltene Stadt, Stuttgart ${ }^{3} 1961$.

Großbölting, Thomas: Der verlorene Himmel. Glaube in Deutschland seit 1945, Bonn 2013.

Halbrock, Christian: „Basisarbeit mit der kirchlichen Jugend und Ausbesserungen am Kirchendach. Die Ost-West-Treffen der evangelischen Kirche in der DDR", in: Deutschland-Archiv 4 (2011), S. 536-545.

Härtel, Christian / Kabus, Petra (Hrsg.): Das Westpaket. Geschenksendung, keine Handelsware, Berlin 2000.

Hefft, Gesine: Was eint uns, wenn uns nichts mehr trennt? Kirchliche Gemeindepartnerschaften vor und nach 1989, Berlin 1996.

Hogebrink, Laurens: „Die armen Verwandten - oder der bessere Teil Deutschlands? Was haben wir voneinander gelernt, was hat uns an den DDR-Kirchen beeindruckt oder irritiert?“, in: epd Dokumentation 14/15 (2013), S. 27-31.

Homeyer, Josef: „Die katholische Kirche im geteilten Deutschland und ihre Bedeutung für das Zusammengehörigkeitsgefühl“, in: Hehl, Ulrich von / Hockerts, Hans Günter: Der Katholizismus - gesamtdeutsche Klammer in den Jahrzehnten der Teilung? Erinnerungen und Berichte, Paderborn u. a. 1996, S. 24-25.

Kleßmann, Christoph: „Verflechtung und Abgrenzung - Umrisse einer gemeinsamen deutschen Nachkriegsgeschichte“, in: Schönhoven, Klaus / Staritz, Dietrich (Hrsg.): Sozialismus und Kommunismus im Wandel. Hermann Weber zum 75. Geburtstag, Köln 1993, S. 486-499.

Kleßmann, Christoph: Die doppelte Staatsgründung. Deutsche Geschichte 1945-1955, Göttingen / Bonn 1982.

Knauft, Wolfgang: Katholische Kirche in der DDR. Gemeinden in der Bewährung. 1945 1980, 2. verbesserte und erweiterte Auflage, Mainz 1980.

Kunter, Katharina: „Zwischen Antikommunismus und ökumenischer Idealisierung. Prägende Orte und Milieus für das DDR-Kirchenbild im Westen“, in: epd Dokumentation 14/15 (2013), S. 36.

Lemke, Michael (Hrsg.): Schaufenster der Systemkonkurrenz. Die Region Berlin-Brandenburg im Kalten Krieg, Köln u. a. 2006.

Lepp, Claudia: Tabu der Einheit? Die Ost-West-Gemeinschaft evangelischer Christen und die deutsche Teilung (1945-1969), Göttingen 2005.

Maser, Peter: „Die evangelischen Kirchen in der DDR aus der Perspektive des Westens. Blicke über den Zaun - von der Bundesrepublik aus“, in: epd Dokumentation 14/15 (2013), S. 17.

Maser, Peter: Die Kirchen in der DDR, Bonn 2000.

Maser, Peter: Kirchen und Religionsgemeinschaften in der DDR 1949-1989. Ein Rückblick auf vierzig Jahre in Daten, Fakten und Meinungen, Konstanz 1992.

Meckel, Markus: „Geborgenheit und Wagnis“, in: Kleßmann, Christoph (Hrsg.): Kinder der Opposition. Berichte aus Pfarrhäusern in der DDR, Gütersloh 1993, S. 101.

Plato, Alexander von: „Interview-Richtlinien“, in: Leh, Almut / Plato, Alexander von / Thonfeld, Christoph (Hrsg.): Hitlers Sklaven. Lebensgeschichtliche Analysen zur Zwangsarbeit im internationalen Vergleich, Wien u. a. 2008, S. 443-450.

Plume, Paul: Briefe in die Freiheit 1988-1990, Ahrensfelde 2009, unveröffentlicht. 
Puschmann, Hellmut: „Zur Brückenfunktion des Deutschen Caritasverbandes“, in: von Hehl, Ulrich / Hockerts Hans Günter: Der Katholizismus - gesamtdeutsche Klammer in den Jahrzehnten der Teilung? Erinnerungen und Berichte, Paderborn u. a. 1996, S. 129.

Rittberger-Klas, Karoline: Kirchenpartnerschaften im geteilten Deutschland. Am Beispiel der Landeskirchen Württemberg und Thüringen, Göttingen 2006.

Seidel, J. Jürgen: „Neubeginn“ in der Kirche? Die evangelischen Landes- und Provinzialkirchen in der SBZ/DDR im gesellschaftspolitischen Kontext der Nachkriegszeit 1945-1953, Göttingen 1989.

Sell, Helmut: „Rückschau auf 25 Jahre Partnerschaft“, in: Partnerschaft der Kirchengemeinden Kirn und St. Petri Luckenwalde im geteilten Deutschland von 1978 bis 2004, Broschüre, S. 5.

Silomon, Anke: Anspruch und Wirklichkeit der ,,besonderen Gemeinschaft“. Der OstWest-Dialog der deutschen evangelischen Kirche 1969-1991, Göttingen 2006.

Wanke, Joachim: „Bemühungen um den kirchlichen Zusammenhalt zwischen Ost und West", in: von Hehl, Ulrich / Hockerts, Hans Günter: Der Katholizismus - gesamtdeutsche Klammer in den Jahrzehnten der Teilung? Erinnerungen und Berichte, Paderborn u. a. 1996, S. 36.

Winter, Friedrich: „Kirchliche Wege zwischen Ost und West (1949-1989). Hinweise auf eine dringende Forschungsaufgabe“, in: Hoburg, Ralf (Hrsg.): Unter dem Dach der Kirche. Festschrift für Joachim Rogge zum 70. Geburtstag, Leipzig 1999, S. 146-147.

Zeh, Michael: „Luckenwalde - Dank für eine Partnerschaft“, in: Partnerschaft der Kirchengemeinden Kirn und St. Petri Luckenwalde im geteilten Deutschland von 1978 bis 2004, Broschüre, S. 22.

\section{Internetquellen}

Przybilski, Helmut: Erfahrungen mit Ost-West-Partnerschaften, Vortrag, online abrufbar unter: https:/www.eaberlin.de/nachlese/chronologisch-nach-jahren/2015/ost-westpartnerschaft/przybilski-helmut-ost-west-partnerschaften-2015.pdf [8.6.2016, 16:53 Uhr].

Schröder, Susanne: Bericht über unsere Gemeindepartnerschaft, Vortrag, online abrufbar unter: https:/www.eaberlin.de/nachlese/chronologisch-nach-jahren/2015/ost-westpartnerschaft/schroeder-susanne-gemeindepartnerschaft-2015.pdf [08.6.2016, 16:30 Uhr].

\section{Daten der Interviews}

Interview mit A.B., 21.1.2016

Interview mit Arnold Löwenbrück, 29.1.2016

Interview mit F.J., 21.6.2017

Interview mit G.S., 24. 6. 2017

Interview mit Paul Plume, 2.2.2016

Interview mit Siegfried Schmidtmann, 2005, Oncken-Archiv, ohne Signatur Interview mit Prälat Roland Steinke, 23.5.2017 
Maria Neumann, Department of History, Humboldt-Universität zu Berlin, Unter den Linden 6, 10099 Berlin, Germany, e-mail: maria.neumann.hu@googlemail.com

Citation: Neumann, Maria: „Der Nächste ist ein Anderer. Grenzübergreifende Gemeindepartnerschaften in Berlin und Brandenburg während des Kalten Kriegs“, in: Kallhoff, Angela / Schulte-Umberg, Thomas (eds.): Moralities of Warfare and Religion (J-RaT 2018 / 1) pp. 224-253.

Datum der Publikation: 16.07.2018 